\title{
Periodic Solutions to Differential Variational Inequalities of Parabolic-elliptic Type
}

\author{
Thi Van Anh Nguyen
}

\begin{abstract}
In this work, we consider a model formulated by a dynamical system and an elliptic variational inequality. We prove the solvability of initial value and periodic problems. Finally, two illustrative examples are given to show the applicability of our theoretical results.
\end{abstract}

\section{Introduction}

Let $\left(X,\|\cdot\|_{X}\right)$ be a Banach space and $\left(Y,\|\cdot\|_{Y}\right)$ be a reflexive Banach space with the dual $Y^{*}$. We consider the following problem

$$
\begin{gathered}
x^{\prime}(t) \in A x(t)+F(t, x(t), y(t)), \quad t>0, \\
B y(t)+\partial \phi(y(t)) \ni h(t, x(t), y(t)), \quad t>0,
\end{gathered}
$$

where $(x(\cdot), y(\cdot))$ takes values in $X \times Y ; \phi: Y \rightarrow(-\infty, \infty]$ is a proper, convex and lower semicontinuous function with the subdifferential $\partial \phi \subset Y \times Y^{*}$. F is a multivalued function defined on $\mathbb{R}^{+} \times X \times Y$. In our system, $A$ is a closed linear operator which generates a $C_{0}$-semigroup in $X ; B: Y \rightarrow Y^{*}$ and $h: \mathbb{R}^{+} \times X \times Y \rightarrow Y^{*}$ are given maps which will be specified in the next sections.

In this paper, we study the existence of a periodic solution for this problem, that is, we prove the solvability of $1.1-1.2$ with $T$-periodic condition

$$
x(t)=x(t+T), \quad \forall t \geq 0
$$

for given $T>0$.

When $F$ and $h$ are autonomous maps, the system $1.1-1.2$ was investigated in [13. In this work, the existence of solutions and the existence of a global attractor for $m$ semiflow generated by solution set were proved. The application of such parabolic-elliptic system has been employed to model different problems appeared in many recent works, for

Received October 16, 2019; Accepted March 5, 2020.

Communicated by Cheng-Hsiung Hsu.

2010 Mathematics Subject Classification. 34A60, 34C25, 35R45, 47H08, 47H10.

Key words and phrases. elliptic variational inequalities, periodic solutions, differential inclusions, measure of noncompactness, fixed point theorems. 
instance, in study the motion of bacteria by chemotaxic (e.g., 7]) and in image inpainting (e.g., [8]).

In the case $\phi=I_{K}$, the indicator function of $K$ with $K$ being a closed convex subset in $Y$, namely,

$$
I_{K}(x)= \begin{cases}0 & \text { if } x \in K \\ +\infty & \text { otherwise }\end{cases}
$$

the problem $(1.1-1.2$ reads as follows:

$$
\begin{cases}x^{\prime}(t)=A x(t)+F(t, x(t), y(t)), & t>0, \\ y(t) \in K, & \forall t \geq 0, \\ \langle B y(t), z-y(t)\rangle \geq\langle h(t, x(t), y(t)), z-y(t)\rangle, & \forall z \in K, t>0,\end{cases}
$$

where $\langle\cdot, \cdot\rangle$ stands for the duality pairing between $Y^{*}$ and $Y$. In the latter model, we have to mention that, when $X=\mathbb{R}^{n}, Y=\mathbb{R}^{m}$ and $F$ is single-valued, the problem (1.3) becomes differential variational inequality studied by Anh and Ke [12], Liu, Loi and Obukhovskii [11, Pang and Stewart [14, etc. Differential variational inequalities in finite dimensional spaces were a new modeling paradigm of variational analysis to treat many applied dynamical problems in engineering, operations research, and physical sciences such as mechanical impact problems, electrical circuits with ideal diodes, Coulomb friction problems for contacting bodies, economical dynamics. For more details, one can refer the interested reader to [5, 15] and references therein.

Considering (1.1)-(1.2) as a differential variational inequality associated with a convex subdifferential, we aim at studying the solvability of initial value and periodic problems. More precisely, we consider the periodic problems for semilinear systems described by parabolic-elliptic differential variational inequalities with both single-valued and multivalued perturbations. Based on the techniques presented by Bothe in [3] and some fixed point theorems, we treat $1.1-(1.2)$ in the case that the semigroup generated by $A$ is norm continuous and exponentially stable in single-valued situations, adding the compactness condition in multivalued situations. Our motivation for the present work is that, up to our knowledge, no attempt has been made to establish the results concerning initial value and periodic problems for the general model of differential equations/inclusions mixed elliptic variational inequalities $1.1-1.2$.

The paper is organized as follows. In the next section, we recall some notions and facts related to measures of noncompactness, multivalued analysis. Section 3 is devoted to the existence and uniqueness results of the problem $(1.1)-(1.2)$ with periodic condition in case of single valued perturbations. In Section 4, we prove that there exists a periodic solution of $1.1-1.2$ in multivalued cases. In Section 5 we give two examples, which illustrate 
the obtained results for partial differential equations/inclusions with obstacle constraints and lattice differential variational inequalities.

\section{Preliminaries}

\subsection{Measure of non-compactness}

Let $E$ be a Banach space. Denote

$$
\mathcal{P}(E)=\{B \subset E: B \neq \emptyset\} \text { and } \mathcal{B}(E)=\{B \in \mathcal{P}(E): B \text { is bounded }\} .
$$

We give here the definition of measure of noncompactness (see [1]).

Definition 2.1. A function $\beta: \mathcal{B}(E) \rightarrow \mathbb{R}^{+}$is called a measure of noncompactness (MNC) in $E$ if

$$
\beta(\overline{\mathrm{co}} \Omega)=\beta(\Omega) \quad \text { for every } \Omega \in \mathcal{B}(E),
$$

where $\overline{\mathrm{co}} \Omega$ is the closure of the convex hull of $\Omega$. An MNC $\beta$ is called

(i) monotone if $\Omega_{0}, \Omega_{1} \in \mathcal{B}(E), \Omega_{0} \subset \Omega_{1}$ implies $\beta\left(\Omega_{0}\right) \subset \beta\left(\Omega_{1}\right)$;

(ii) nonsingular if $\beta(\{a\} \cup \Omega)=\beta(\Omega)$ for any $a \in E, \Omega \in \mathcal{B}(E)$;

(iii) invariant with respect to union with compact set if $\beta(K \cup \Omega)=\beta(\Omega)$ for every relatively compact set $K \subset E$ and $\Omega \in \mathcal{B}(E)$;

(iv) algebraically semi-additive if $\beta\left(\Omega_{0}+\Omega_{1}\right) \leq \beta\left(\Omega_{0}\right)+\beta\left(\Omega_{1}\right)$ for any $\Omega_{0}, \Omega_{1} \in \mathcal{B}(E)$;

(v) regular if $\beta(\Omega)=0$ is equivalent to the relative compactness of $\Omega$.

An important example of MNC is the Hausdorff MNC $\chi(\cdot)$, which is defined as follows:

$$
\chi(\Omega)=\inf \{\epsilon: \Omega \text { has a finite } \epsilon \text {-net }\} .
$$

In particular, it is known that the Hausdorff $\mathrm{MNC}$ on $C([\theta, T] ; E)$ given by (see [1])

$$
\chi_{T}(D)=\lim _{\delta \rightarrow 0} \sup _{x \in D} \max _{\substack{t, s \in[\theta, T] \\|t-s|<\delta}}\|x(t)-x(s)\|+\sup _{t \in[\theta, T]} e^{-L t} \chi(D(t)) .
$$

Based on Hausdorff MNC $\chi$, one can define the sequential $M N C \chi_{0}$ as follows:

$$
\chi_{0}(\Omega)=\sup \{\chi(D): D \in \Delta(\Omega)\}
$$

where $\Delta(\Omega)$ is the collection of all at-most-countable subset of $\Omega$ (see $[1]$ ). We know that

$$
\frac{1}{2} \chi(\Omega) \leq \chi_{0}(\Omega) \leq \chi(\Omega)
$$

for all bounded set $\Omega \subset E$. Then the following property is evident. 
Proposition 2.2. Let $\chi$ be the Hausdorff $M N C$ in $E$ and $\Omega \subset E$ be a bounded set. Then for every $\epsilon>0$, there exists a sequence $\left\{x_{n}\right\} \subset \Omega$ such that

$$
\chi(\Omega) \leq 2 \chi\left(\left\{x_{n}\right\}\right)+\epsilon .
$$

If $\Omega \subset L^{1}(J ; E)$ such that, for all $f \in \Omega,\|f(t)\| \leq \nu(t)$ for a.e. $t \in J$, where $\nu \in$ $L^{1}(J):=L^{1}(J ; \mathbb{R})$, then we say that $\Omega$ is integrably bounded.

Proposition 2.3. [9] If $\left\{w_{n}\right\} \subset L^{1}([0, T] ; E)$ is integrably bounded, then we have

$$
\chi\left(\left\{\int_{0}^{t} w_{n}(s) d s\right\}\right) \leq 2 \int_{0}^{t} \chi\left(\left\{w_{n}(s)\right\}\right) d s
$$

for $t \in[0, T]$.

Using Propositions 2.2 and 2.3, we have the following statement [10, Proposition 2.5].

Proposition 2.4. Let $D \subset L^{1}(0, T ; E)$ such that

(1) $\|\xi(t)\| \leq \nu(t)$ for all $\xi \in D$ and for a.e. $t \in[0, T]$,

(2) $\chi(D(t)) \leq q(t)$ for a.e. $t \in[0, T]$,

where $\nu, q \in L^{1}(0, T ; \mathbb{R})$. Then

$$
\chi\left(\int_{0}^{t} D(s) d s\right) \leq 4 \int_{0}^{t} q(s) d s,
$$

here $\int_{0}^{t} D(s) d s=\left\{\int_{0}^{t} \xi(s) d s: \xi \in D\right\}$.

\subsection{Set-valued analysis}

We make use of some notions of set-valued analysis. Let $Y$ be a metric space, and $\mathcal{P}(E)$ be the collection of all nonempty subsets of $E$.

Definition 2.5. A multi-valued map (multimap) $\mathcal{F}: Y \rightarrow \mathcal{P}(E)$ is said to be:

(i) upper semi-continuous (u.s.c.) if $\mathcal{F}^{-1}(V)=\{y \in Y: \mathcal{F}(y) \cap V \neq \emptyset\}$ is a closed subset of $Y$ for every closed set $V \subset E$;

(ii) weakly upper-semicontinuous (weakly u.s.c.) if $\mathcal{F}^{-1}(V)$ is closed subset of $Y$ for all weakly closed set $V \subset E$;

(iii) closed if its graph $\Gamma_{\mathcal{F}}=\{(y, z): z \in \mathcal{F}(y)\}$ is a closed subset of $Y \times E$;

(iv) compact if its range $\mathcal{F}(Y)$ is relatively compact in $E$; 
(v) quasi-compact if its restriction to any compact subset $A \subset Y$ is compact.

The following results will be used.

Lemma 2.6. [9, Theorem 1.1.12] Let $G: Y \rightarrow \mathcal{P}(E)$ be a closed quasi-compact multimap with compact values. Then $G$ is u.s.c.

Lemma 2.7. [3, Proposition 2] Let $E$ be a Banach space and $\Omega$ be a nonempty subset of another Banach space. Assume that $G: \Omega \rightarrow \mathcal{P}(E)$ is a multimap with weakly compact, convex values. Then $G$ is weakly u.s.c. if and only if $\left\{x_{n}\right\} \subset \Omega$ with $x_{n} \rightarrow x_{0} \in \Omega$ and $y_{n} \in G\left(x_{n}\right)$ implies $y_{n} \rightarrow y_{0} \in G\left(x_{0}\right)$ up to a subsequence.

Definition 2.8. A subset $D \in L^{1}(0, T ; X)$ is said to be semicompact if it is integrably bounded and the set $D(t)=\{f(t): f \in D\}$ is relatively compact in $X$ for a.e. $t \in[0, T]$.

We known that if a sequence $\left\{f_{n}\right\} \subset L^{1}(0, T ; X)$ is semicompact, then it is weakly compact (e.g., [9]). We recall some well-known fixed point theorems for multivalued maps.

Theorem 2.9. Let $E$ be a Banach space and $D \subset E$ be a nonempty compact convex subset. If the multimap $F: D \rightarrow \mathcal{P}(D)$ has closed graph with convex values, then $\mathcal{F}$ has a fixed point.

Theorem 2.10. Let $\mathcal{M}$ be a bounded convex and closed subset of a Banach space $E$, and let $\mathcal{F}: \mathcal{M} \rightarrow \mathcal{P}(\mathcal{M})$ be a compact, u.s.c. multimap with compact convex values. Then $\operatorname{Fix}(\mathcal{F}):=\{x \in E: x \in \mathcal{F}(x)\}$ is a nonempty compact set.

\section{In case of single-valued perturbation}

In this section, we consider the system $1.1-1.2$ when $F$ is a single valued map. By some suitable hypotheses imposed on given functions, we obtain the results concerning the solvability of 1.1 - 1.2 under initial value condition and periodic condition. Particularly, we consider the problem as follows:

$$
\begin{gathered}
x^{\prime}(t)=A x(t)+F(t, x(t), y(t)), \\
y^{\prime}(t)+B y(t)+\partial(y(t)) \ni h(t, x(t), y(t)) .
\end{gathered}
$$

3.1. The existence of solution with initial condition

We consider differential variational inequality $(3.1)-(3.2)$ with initial datum

$$
x(0)=x_{0} .
$$

To get the solvability results, we need the following assumptions. 
(A) $A$ is a closed linear operator generating a $C_{0}$-semigroup $(S(t))_{t \geq 0}$ in $X$.

(B) $B$ is a linear continuous operator from $Y$ to $Y^{*}$ defined by

$$
\langle u, B v\rangle=b(u, v), \quad \forall u, v \in Y,
$$

where $b: Y \times Y \rightarrow \mathbb{R}$ is a bilinear continuous function on $Y \times Y$ such that

$$
b(u, u) \geq \eta_{B}\|u\|_{Y}^{2}
$$

(F) $F: \mathbb{R}^{+} \times X \times Y \rightarrow X$ satisfies

$$
\left\|F(t, x, y)-F\left(t, x^{\prime}, y^{\prime}\right)\right\|_{X} \leq a(t)\left\|x-x^{\prime}\right\|_{X}+b(t)\left\|y-y^{\prime}\right\|_{Y}
$$

where $a, b \in L_{\text {loc }}^{1}\left(\mathbb{R}^{+} ; \mathbb{R}^{+}\right)$.

(H) $h: \mathbb{R}^{+} \times X \times Y \rightarrow Y^{*}$ is a Lipschitz continuous map. In particular, there exist two positive constants $\eta_{1 h}, \eta_{2 h}$ and a positive continuous function $\eta_{h}(\cdot, \cdot), \eta_{h}(t, t)=0$ such that

$$
\left\|h\left(t, x_{1}, u_{1}\right)-h\left(t_{1}, x_{2}, u_{2}\right)\right\|_{*} \leq \eta_{h}\left(t, t_{1}\right)+\eta_{1 h}\left\|x_{1}-x_{2}\right\|_{X}+\eta_{2 h}\left\|u_{1}-u_{2}\right\|_{Y}
$$

for all $t \in \mathbb{R}^{+}, x_{1}, x_{2} \in X ; u_{1}, u_{2} \in Y$, where $\|\cdot\|_{*}$ is the norm in dual space $Y^{*}$.

Letting $T>0$, we mention here the definition of a mild solution of the problem $3.1-$ (3.3).

Definition 3.1. A pair of continuous functions $(x, y)$ is said to be a mild solution of (3.1) -3.3 on $[0, T]$ if

$$
\begin{gathered}
x(t)=S(t) x_{0}+\int_{0}^{t} S(t-s) F(t, x(s), y(s)) d s, \quad t \in[0, T], \\
B y(t)+\partial \phi(y(t)) \ni h(t, x(t), y(t)), \quad \forall z \in Y, \text { a.e. } t \in(0, T) .
\end{gathered}
$$

We firstly are concerned with the elliptic variational inequality $(1.2)$. Consider the $\operatorname{EVI}(g)$ problem: find $y \in X$ with given $g \in Y^{*}$ satisfying

$$
B y+\partial \phi(y) \ni g
$$

We recall a remarkable result which can be seen in [2] or in [4].

Lemma 3.2. If $B$ satisfies (B) and $g \in X^{*}$, then the solution of (3.4) is unique. Moreover, the corresponding

$$
\mathbb{S}: Y^{*} \rightarrow Y, \quad g \mapsto y
$$

is Lipschitzian. 
Proof. By [2, Theorem 2.3], we obtain that the solution of (3.4) is unique. In order to prove the map $g \rightarrow y$ is Lipschitz continuous from $Y^{*}$ to $Y$, let $y_{1}, y_{2}$ be the solutions of two elliptic variational inequalities with respect to given data $g_{1}, g_{2}$, namely,

$$
B y_{1}+\partial \phi\left(y_{1}\right) \ni g_{1}, \quad B y_{2}+\partial \phi\left(y_{2}\right) \ni g_{2},
$$

or equivalent to

$$
\begin{array}{ll}
b\left(y_{1}, y_{1}-v\right)+\phi\left(y_{1}\right)-\phi(v) \leq\left\langle y_{1}-v, g_{1}\right\rangle, & \forall v \in Y \\
b\left(y_{2}, y_{2}-v\right)+\phi\left(y_{2}\right)-\phi(v) \leq\left\langle y_{2}-v, g_{2}\right\rangle, & \forall v \in Y .
\end{array}
$$

Choosing $v=y_{2}$ and replacing to (3.5), choosing $v=y_{1}$ and replacing to (3.6), then combining them, we have

$$
b\left(y_{1}-y_{2}, y_{1}-y_{2}\right) \leq\left\langle y_{1}-y_{2}, g_{1}-g_{2}\right\rangle .
$$

Hence,

$$
\left\|y_{1}-y_{2}\right\|_{Y} \leq \frac{1}{\eta_{B}}\left\|g_{1}-g_{2}\right\|_{*}
$$

or

$$
\left\|\mathbb{S}\left(g_{1}\right)-\mathbb{S}\left(g_{2}\right)\right\|_{Y} \leq \frac{1}{\eta_{B}}\left\|g_{1}-g_{2}\right\|_{*}
$$

thanks to (B). The lemma is proved.

Now, for each $(\tau, x) \in \mathbb{R}^{+} \times X$, consider the original form of 1.2

$$
B y+\partial \phi(y) \ni h(\tau, x, y)
$$

Using the last lemma, we obtain the following existence result and property of solution map for 3.8 .

Lemma 3.3. Let $(\mathrm{B})$ and $(\mathrm{H})$ hold. In addition, suppose that $\eta_{B}>\eta_{2 h}$. Then for each $(\tau, x) \in \mathbb{R}^{+} \times X$, there exists a unique solution $y \in Y$ of $(3.8)$. Moreover, the solution mapping

$$
\mathbb{V} \mathbb{I}:[0, \infty) \times X \rightarrow Y, \quad(\tau, x) \mapsto y
$$

is Lipchizian, more precisely

$$
\left\|\mathbb{V} \mathbb{I}\left(\tau, x_{1}\right)-\mathbb{V} \mathbb{I}\left(\tau, x_{2}\right)\right\|_{Y} \leq \frac{\eta_{1 h}}{\eta_{B}-\eta_{2 h}}\left\|x_{1}-x_{2}\right\|_{X}
$$


Proof. Let $(\tau, x) \in \mathbb{R}^{+} \times X$ be fixed. We consider the composition map $\mathbb{S} \circ h(\tau, x, \cdot): Y \rightarrow$ $Y$. Employing (3.7), we have

$$
\begin{aligned}
\left\|\mathbb{S}\left(h\left(\tau, x, y_{1}\right)\right)-\mathbb{S}\left(h\left(\tau, x, y_{2}\right)\right)\right\|_{Y} & \leq \frac{1}{\eta_{B}}\left\|h\left(\tau, x, y_{1}\right)-h\left(\tau, x, y_{2}\right)\right\|_{*} \\
& \leq \frac{\eta_{2 h}}{\eta_{B}}\left\|y_{1}-y_{2}\right\|_{Y} .
\end{aligned}
$$

Because $\eta_{2 h}<\eta_{B}$ and $y \mapsto \mathbb{S}(h(\tau, x, \cdot))$ is a contraction map, $\mathbb{S} \circ h(\tau, x, \cdot)$ admits a unique fixed point, which is a unique solution of (3.8).

It remains to show that the map $(\tau, x) \mapsto y$ is Lipschitz continuous with respect to the second variable. Let $\mathbb{V} \mathbb{I}\left(\tau, x_{1}\right)=y_{1}, \mathbb{V} \mathbb{I}\left(\tau, x_{2}\right)=y_{2}$. Then, one has

$$
\begin{aligned}
\left\|y_{1}-y_{2}\right\|_{Y} & =\left\|\mathbb{S}\left(h\left(\tau, x_{1}, y_{1}\right)\right)-\mathbb{S}\left(h\left(\tau, x_{2}, y_{2}\right)\right)\right\|_{Y} \\
& \leq \frac{1}{\eta_{B}}\left\|h\left(\tau, x_{1}, y_{1}\right)-h\left(\tau, x_{2}, y_{2}\right)\right\|_{*} \\
& \leq \frac{\eta_{1 h}}{\eta_{B}}\left\|x_{1}-x_{2}\right\|_{X}+\frac{\eta_{2 h}}{\eta_{B}}\left\|y_{1}-y_{2}\right\|_{Y} .
\end{aligned}
$$

Therefore,

$$
\left\|y_{1}-y_{2}\right\|_{Y} \leq \frac{\eta_{1 h}}{\eta_{B}-\eta_{2 h}}\left\|x_{1}-x_{2}\right\|_{X}
$$

which leads to the conclusion of lemma.

In order to solve (1.1)-(1.2), we convert it to a differential equation. We consider the following map:

$$
G(t, x):=F(t, x, \mathbb{V} \mathbb{I}(t, x)), \quad(t, x) \in \mathbb{R}^{+} \times X .
$$

One sees that $G: \mathbb{R}^{+} \times X \rightarrow X$. Moreover, by assumption $(\mathrm{F})$ and the continuity of $\mathbb{V} \mathbb{I}$, we deduce that the map $G(t, \cdot)$ is continuous for each $t \geq 0$. By the estimate (3.9), and the Hausdorff MNC property, one has

$$
\chi_{Y}(\mathbb{V} \mathbb{I}(t, \Omega)) \leq \frac{\eta_{1 h}}{\eta_{B}-\eta_{2 h}} \chi_{X}(\Omega)
$$

where $\chi_{Y}$ is the Hausdorff MNC in $Y$. In the case the semigroup $S(\cdot)$ is non-compact, we have

$$
\begin{aligned}
\chi_{X}(G(t, \Omega)) & =\chi_{X}(F(t, \Omega, \mathbb{V} \mathbb{I}(t, \Omega))) \leq a(t) \chi_{X}(\Omega)+b(t) \chi_{Y}(\mathbb{V} \mathbb{I}(t, \Omega)) \\
& \leq a(t) \chi_{X}(\Omega)+b(t)\left(\frac{\eta_{1 h}}{\eta_{B}-\eta_{2 h}} \chi_{X}(\Omega)\right) \leq\left(a(t)+\frac{b(t) \eta_{1 h}}{\eta_{B}-\eta_{2 h}}\right) \chi_{X}(\Omega) \\
& =p_{G}(t) \chi_{X}(\Omega),
\end{aligned}
$$

where $p_{G}(t)=\left(a(t)+\frac{b(t) \eta_{1 h}}{\eta_{B}-\eta_{2 h}}\right)$. 
Concerning the growth condition of $G$, by (F2) we arrive at

$$
\begin{aligned}
\|G(t, x)\|_{X} & \leq a(t)\|x\|_{X}+b(t)\|\mathbb{V} \mathbb{I}(t, x)\|_{Y}+\|F(t, 0,0)\|_{X} \\
& \leq a(t)\|x\|_{X}+b(t) \frac{\eta_{1 h}}{\eta_{B}-\eta_{2 h}}\|x\|_{X}+\|\mathbb{V} \mathbb{I}(t, 0)\|_{Y}+\|F(t, 0,0)\|_{X} .
\end{aligned}
$$

By a process similar to that in Lemma 3.3 , we obtain

$$
\|\mathbb{V} \mathbb{I}(t, x)\| \leq \frac{\eta_{h}(t, 0)}{\eta_{B}-\eta_{2 h}}+\frac{\eta_{1 h}}{\eta_{B}-\eta_{2 h}}\|x\|+\|\mathbb{V} \mathbb{I}(0,0)\| .
$$

Thus, we obtain

$$
\|G(t, x)\|_{X} \leq \eta_{G}(t)\|x\|_{X}+d(t)
$$

where $\eta_{G}(t):=\left(a(t)+\frac{b(t) \eta_{1 h}}{\eta_{B}-\eta_{2 h}}\right)$ and $d(t)=\frac{\eta_{h}(t, 0)}{\eta_{B}-\eta_{2 h}}+\|\mathbb{V} \mathbb{I}(0,0)\|+\|F(t, 0,0)\|_{X}$. In addition, we also get that

$$
\begin{aligned}
\left\|G(t, x)-G\left(t, x^{\prime}\right)\right\|_{X} & =\left\|F(t, x, \mathbb{V} \mathbb{I}(t, x))-F\left(t, x^{\prime}, \mathbb{V} \mathbb{I}\left(t, x^{\prime}\right)\right)\right\|_{X} \\
& \leq a(t)\left\|x-x^{\prime}\right\|_{X}+b(t)\left\|\mathbb{V} \mathbb{I}(t, x)-\mathbb{V} \mathbb{I}\left(t, x^{\prime}\right)\right\|_{Y} \\
& \leq a(t)\left\|x-x^{\prime}\right\|_{X}+\frac{b(t) \eta_{1 h}}{\eta_{B}-\eta_{2 h}}\left\|x-x^{\prime}\right\|_{X} \\
& \leq\left(a(t)+\frac{b(t) \eta_{1 h}}{\eta_{B}-\eta_{2 h}}\right)\left\|x-x^{\prime}\right\|_{X} \\
& \leq \gamma(t)\left\|x-x^{\prime}\right\|_{X}
\end{aligned}
$$

where $\gamma(t)=\left(a(t)+\frac{b(t) \eta_{1 h}}{\eta_{B}-\eta_{2 h}}\right)$.

By the aforementioned setting, the problem $3.1-(3.2)$ is converted to

$$
x^{\prime}(t)-A x(t)=G(t, x(t)), \quad t \in[0, T] .
$$

Now we see that, a pair of functions $(x, y)$ solution of $3.1-3.2$ with initial value $x(0)=x_{0}$ if and only if

$$
\begin{aligned}
& x(t)=S(t) x_{0}+\int_{0}^{t} S(t-s) G(s, x(s)) d s, \quad t \in[0, T], \\
& y(t)=\mathbb{V} \mathbb{I}(t, x(t)) .
\end{aligned}
$$

Consider the Cauchy operator

$$
\mathcal{W}: L^{1}(0, T, X) \rightarrow C([0, T] ; X), \quad \mathcal{W}(f)(t)=\int_{0}^{t} S(t-s) f(s) d s .
$$

For a given $x_{0} \in X$, we introduce the mild solution operator

$$
\mathcal{F}: C([0, T] ; X) \rightarrow C([0, T] ; X), \quad \mathcal{F}(x)=S(\cdot) x_{0}+\mathcal{W}(G(\cdot, x(\cdot))) .
$$

It is evident that $x$ is a fixed point of $\mathcal{F}$ if and only if $x$ is the first component of the mild solution of (3.1)-(3.3). In order to prove the existence result for problem (3.1)-(3.3), we make use of the Schauder fixed point theorem. 
Lemma 3.4. Let $E$ be a Banach space and $D \subset E$ be a nonempty compact convex subset. If the map $\mathcal{F}: D \rightarrow D$ is continuous, then $\mathcal{F}$ has a fixed point.

We have the following result related to the operator $\mathcal{W}$ (see $[9$ ).

Proposition 3.5. Let (A) hold. If $D \subset L^{1}(0, T ; X)$ is semicompact, then $\mathcal{W}(D)$ is relatively compact in $C(J ; X)$. In particular, if sequence $\left\{f_{n}\right\}$ is semicompact and $f_{n} \rightarrow f^{*}$ in $L^{1}(0, T ; X)$ then $\mathcal{W}\left(f_{n}\right) \rightarrow \mathcal{W}\left(f^{*}\right)$ in $C([0, T] ; X)$.

Theorem 3.6. Let the hypotheses (A), (B), (F) and (H) hold. Then the problem (3.1)(3.3) has at least one mild solution $(x(\cdot), y(\cdot))$ for given $x_{0} \in X$.

Proof. We now show that there exists a nonempty convex subset $\mathcal{M}_{0} \subset C([0, T] ; X)$ such that $\mathcal{F}\left(\mathcal{M}_{0}\right) \subset \mathcal{M}_{0}$.

Let $z=F(x)$, then we have

$$
\begin{aligned}
\|z(t)\|_{X} & \leq\left\|S(t) x_{0}\right\|_{X}+\left\|\int_{0}^{t} S(t-s) G(s, x(s)) d s\right\|_{X} \\
& \leq M\left\|x_{0}\right\|_{X}+\int_{0}^{t}\|S(t-s)\|_{\mathcal{L}(X)}\|\| G(s, x(s)) \|_{X} d s \\
& \leq M\left\|x_{0}\right\|_{X}+M \int_{0}^{t}\left(\eta_{G}(s)\|x(s)\|_{X}+d(s)\right) d s,
\end{aligned}
$$

where $M=\sup \left\{\|S(t)\|_{\mathcal{L}(X)}: t \in[0, T]\right\}$.

Denote

$$
\mathcal{M}_{0}=\left\{x \in C([0, T] ; X):\|x(t)\|_{X} \leq \kappa(t), \forall t \in[0, T]\right\},
$$

where $\kappa$ is the unique solution of the integral equation

$$
\kappa(t)=M\left\|x_{0}\right\|_{X}+M \int_{0}^{t}\left(\eta_{G}(s) \kappa(s)+d(s)\right) d s .
$$

It is obvious that $\mathcal{M}_{0}$ is a closed, convex subset of $C([0, T] ; X)$ and $\mathcal{F}\left(\mathcal{M}_{0}\right) \subset \mathcal{M}_{0}$. Set

$$
\mathcal{M}_{k+1}=\overline{\operatorname{co}} \mathcal{F}\left(\mathcal{M}_{k}\right), \quad k=0,1,2, \ldots
$$

here, the notation $\overline{\mathrm{co}}$ stands for the closure of convex hull of a subset in $C([0, T] ; X)$. We see that $\mathcal{M}_{k}$ is a closed convex set and $\mathcal{M}_{k+1} \subset \mathcal{M}_{k}$ for all $k \in \mathbb{N}$.

Let $\mathcal{M}=\bigcap_{k=0}^{\infty} \mathcal{M}_{k}$. In order to get $\mathcal{M} \neq \emptyset$, we will first show that $\mu_{k}(t)=$ $\chi_{X}\left(\mathcal{M}_{k}(t)\right) \rightarrow 0$ as $k \rightarrow \infty$.

If $\{S(t)\}$ is a compact semigroup, we get $\mu_{k}(t)=0, \forall t \geq 0$. On the other hand, if $\{S(t)\}$ is noncompact, we have

$$
\begin{aligned}
\mu_{k+1}(t) & \leq \chi_{X}\left(\int_{0}^{t} S(t-s) G\left(s, \mathcal{M}_{k}(s)\right) d s\right) \\
& \leq 4 M \int_{0}^{t} \chi_{X}\left(G\left(s, \mathcal{M}_{k}(s)\right)\right) d s \leq 4 M \int_{0}^{t} p_{G}(s) \chi\left(\mathcal{M}_{k}(s)\right) d s .
\end{aligned}
$$


Hence,

$$
\mu_{k+1}(t) \leq 4 M \int_{0}^{t} p_{G}(s) \mu_{k}(s) d s .
$$

Putting $\mu_{\infty}(t)=\lim _{k \rightarrow \infty} \mu_{k}(t)$ and passing to the limit, we obtain

$$
\mu_{\infty}(t) \leq 4 M \int_{0}^{t} p_{G}(s) \mu_{\infty}(s) d s .
$$

By using the Gronwall inequality, it follows that $\mu_{\infty}(t)=0$ for all $t \in J$.

Now, let $x_{k} \in \mathcal{M}_{k}, k \geq 1$ as follows:

$$
x_{0}=0 \in \mathcal{M}_{0}, \quad x_{1}=\mathcal{F}\left(x_{0}\right), \quad \ldots, \quad x_{k+1}=\mathcal{F}\left(x_{k}\right) .
$$

Then, we have

$$
\begin{gathered}
\chi\left(\left\{x_{k}(t): k \in \mathbb{N}\right\}\right)=\chi\left(\left\{x_{k}(t): k \in \mathbb{N}, k \geq N\right\}\right), \quad \forall N \in \mathbb{N}, \\
\chi\left(\left\{x_{k}(t): k \in \mathbb{N}, k \geq N\right\}\right) \leq \chi\left(\mathcal{M}_{N}(t)\right) \rightarrow 0 \quad \text { as } N \rightarrow \infty .
\end{gathered}
$$

Thus,

$$
\chi\left(\left\{x_{k}(t): k \in \mathbb{N}\right\}\right)=0 .
$$

On the other hand, $\left\{x_{k}\right\}$ is integrably bounded due to the integrable boundedness of $\mathcal{M}_{0}$. Then, by applying Proposition $3.5, \mathcal{W}\left(\left\{x_{k}: k \in \mathbb{N}\right\}\right)$ is relatively compact in $C([0, T] ; X)$. We deduce that $\mathcal{F}\left(\left\{x_{k}: k \in \mathbb{N}\right\}\right)=S(t) x_{0}+\mathcal{W}\left(\left\{x_{k}: k \in \mathbb{N}\right\}\right)$ is relatively compact as well. Therefore, one can take a subsequence of $\left\{x_{k}\right\}_{k \geq 1}$ (which is still denoted by $\left\{x_{k}\right\}$ ) such that $x_{k} \rightarrow x^{*}$ as $k \rightarrow \infty$ in $C([0, T] ; X)$. Thus $x^{*} \in \mathcal{M}$, so $\mathcal{M} \neq \emptyset$.

It is easily seen that $\mathcal{M}$ is a closed convex subset of $C([0, T] ; X)$ and $\mathcal{F}(\mathcal{M}) \subset \mathcal{M}$. Moreover, for each $k \geq 0, G\left(s, \mathcal{M}_{k}(s)\right)$ is integrably bounded by the growth property of $G$. Thus, $\mathcal{M}$ is also integrably bounded.

As $\mu_{\infty}(t)=0$ for all $t \in J$, we deduce that $\mathcal{M}(t)$ is relatively compact for all $t \in J$. By the Proposition 3.5, $\mathcal{W}(\mathcal{M})$ is relatively compact in $C([0, T] ; X)$. Then $\mathcal{F}(\mathcal{M})$ is a relatively compact subset in $C([0, T] ; X)$ as well.

Denote

$$
D=\overline{\operatorname{co}} \Phi(\mathcal{M})
$$

It is easy to see that $D$ is a nonempty compact convex subset of $C([0, T] ; X)$ and $\mathcal{F}(D) \subset D$ because $\mathcal{F}(D)=\mathcal{F}(\overline{\mathrm{co}} \mathcal{F}(\mathcal{M})) \subset \mathcal{F}(\mathcal{M}) \subset \overline{\mathrm{co}} \mathcal{F}(\mathcal{M})=D$.

We now consider $\mathcal{F}: D \rightarrow D$. In order to apply the fixed point principle given by Lemma 3.4, one can show that $\mathcal{F}$ is a continuous map. Let $x_{n} \in D$ with $x_{n} \rightarrow x^{*}$ and $y_{n} \in \mathcal{F}\left(x_{n}\right)$ with $y_{n} \rightarrow y^{*}$. Then $y_{n}(t)=S(t) x_{0}+\int_{0}^{t} S(t-s) G\left(s, x_{n}(s)\right) d s$. By the continuity of $G$ we can pass to the limit to get that

$$
x^{*}(t)=S(t) x_{0}+\int_{0}^{t} S(t-s) G\left(s, x^{*}(s)\right) d s .
$$


Then $\mathcal{F}$ has a fixed point $\mathbf{x}$. Therefore, let $\mathbf{y}(\cdot)=\mathbb{V} \mathbb{I}(\cdot, \mathbf{x}(\cdot))$, we conclude that $(\mathbf{x}, \mathbf{y})$ is a mild solution of our problem.

Theorem 3.7. Under the assumptions $(\mathrm{A}),(\mathrm{B}),(\mathrm{F})$ and $(\mathrm{H})$, the system (1.1)-(1.2) has a unique mild solution for each initial datum $x_{0}$.

Proof. Let $\left(x_{1}, y_{1}\right)$ and $\left(x_{2}, y_{2}\right)$ be two mild solutions of $1.1-(1.2), x_{1}(0)=x_{2}(0)=x_{0}$. We have

$$
\begin{aligned}
& x_{1}(t)=S(t) x_{0}+\int_{0}^{t} S(t-s) G\left(s, x_{1}(s)\right) d s, \\
& x_{2}(t)=S(t) x_{0}+\int_{0}^{t} S(t-s) G\left(s, x_{2}(s)\right) d s .
\end{aligned}
$$

Then subtracting two last equations, we have

$$
x_{1}(t)-x_{2}(t)=\int_{0}^{t} S(t-s)\left(G\left(s, x_{1}(s)\right)-G\left(s, x_{2}(s)\right)\right) d s .
$$

By estimate of $G$, we obtain that

$$
\begin{aligned}
\left\|x_{1}(t)-x_{2}(t)\right\|_{X} & \leq \int_{0}^{t}\|S(t-s)\|_{\mathcal{L}(X)}\left\|G\left(s, x_{1}(s)\right)-G\left(s, x_{2}(s)\right)\right\|_{X} d s \\
& \leq M \int_{0}^{t} \gamma(s)\left\|x_{1}(s)-x_{2}(s)\right\|_{X} d s
\end{aligned}
$$

Using the Gronwall inequality, this implies the result of theorem.

\subsection{The existence of mild periodic solution}

In this section, let $T>0$ be a given positive time. We replace $(\mathrm{A}),(\mathrm{F}),(\mathrm{H})$ by the following assumptions:

$\left(\mathrm{A}^{*}\right) A$ satisfies (A) and the semigroup $S(t)$ is exponentially stable with exponent $\alpha$, that is,

$$
\|S(t)\|_{\mathcal{L}(X)} \leq M e^{-\alpha t}, \quad \forall t \geq 0
$$

$\left(\mathrm{F}^{*}\right) F$ satisfies $(\mathrm{F})$ with $a(t) \equiv a$ and $b(t) \equiv b$. Moreover,

$$
F(t, x, y)=F(t+T, x, y), \quad \forall t \geq 0, x \in X, y \in Y
$$

$\left(\mathrm{H}^{*}\right) h$ satisfies $(\mathrm{H})$ and

$$
h(t, x, y)=h(t+T, x, y), \quad \forall t \geq 0, x \in X, y \in Y \text {. }
$$


Definition 3.8. A pair of continuous functions $(x, y)$ is called a mild $T$-periodic solution of $1.1-1.2$ if and only if

$$
\begin{cases}x(t)=S(t-s) x(s)+\int_{s}^{t} S(t-s) F(s, x(s), y(s)) d s, & \forall t \geq s \geq 0, \\ x(t)=x(t+T), & \forall t \geq 0, \\ B y(t)+\partial(\phi(y(t))) \ni h(t, x(t), y(t)) & \text { for a.e. } t \geq 0 .\end{cases}
$$

By Theorem 3.7, due to the unique solvability of (3.11)-3.12, we are in a position to define the following map.

$$
\mathcal{G}: X \rightarrow X, \quad \mathcal{G}\left(x_{0}\right)=S(T) x_{0}+\int_{0}^{T} S(T-s) G(s, x(s)) d s,
$$

where $x$ is a mild solution of $(3.11)$ with $x(0)=x_{0}$.

Theorem 3.9. Under the assumptions $\left(\mathrm{A}^{*}\right),(\mathrm{B}),\left(\mathrm{F}^{*}\right)$ and $\left(\mathrm{H}^{*}\right)$, the system $(1.1)-(1.2)$ has a unique mild T-periodic solution, provided that $\eta_{B}>\eta_{2 h}$ and

$$
\begin{gathered}
\alpha>M\left(a+\frac{b \eta_{1 h}}{\eta_{B}-\eta_{2 h}}\right), \\
M \exp \left(-\left(\alpha-M\left(a+\frac{b \eta_{1 h}}{\eta_{B}-\eta_{2 h}}\right)\right) T\right)<1 .
\end{gathered}
$$

Proof. First of all, we prove that $\mathcal{G}$ has a fixed point. For any $\xi_{1}, \xi_{2} \in X$, let $x_{1}=x_{1}\left(\cdot ; \xi_{1}\right)$, $x_{2}=x_{2}\left(\cdot ; \xi_{2}\right)$ be the mild solutions of 3.11) with initial data $\xi_{1}, \xi_{2}$, respectively. We have

$$
\mathcal{G}\left(\xi_{1}\right)-\mathcal{G}\left(\xi_{2}\right)=S(T)\left(\xi_{1}-\xi_{2}\right)+\int_{0}^{T} S(T-s)\left(G\left(s, x_{1}(s)\right)-G\left(s, x_{2}(s)\right)\right) d s .
$$

By the integral formula of mild solution, one gets

$$
x_{1}(t)-x_{2}(t)=S(t)\left(\xi_{1}-\xi_{2}\right)+\int_{0}^{t} S(t-s)\left(G\left(s, x_{1}(s)\right)-G\left(s, x_{2}(s)\right)\right) d s .
$$

Then employing (3.10, we obtain

$$
\begin{aligned}
& \left\|x_{1}(t)-x_{2}(t)\right\|_{X} \\
\leq & \|S(t)\|_{\mathcal{L}(X)}\left\|\xi_{1}-\xi_{2}\right\|_{X}+\int_{0}^{t}\|S(t-s)\|_{\mathcal{L}(X)}\left\|G\left(s, x_{1}(s)\right)-G\left(s, x_{2}(s)\right)\right\|_{X} d s \\
\leq & M e^{-\alpha t}\left\|\xi_{1}-\xi_{2}\right\|_{X}+M \int_{0}^{t} e^{-\alpha(t-s)} \gamma\left\|x_{1}(s)-x_{2}(s)\right\|_{X} d s,
\end{aligned}
$$

where $\gamma=a+\frac{b \eta_{1 h}}{\eta_{B}-\eta_{2 h}}$. Hence,

$$
e^{\alpha t}\left\|x_{1}(t)-x_{2}(t)\right\|_{X} \leq M\left\|\xi_{1}-\xi_{2}\right\|_{X}+M \gamma \int_{0}^{t} e^{\alpha s}\left\|x_{1}(s)-x_{2}(s)\right\|_{X} d s .
$$


The Gronwall inequality shows that

$$
e^{\alpha t}\left\|x_{1}(t)-x_{2}(t)\right\|_{X} \leq M\left\|\xi_{1}-\xi_{2}\right\|_{X} e^{M \gamma t}
$$

Therefore,

$$
\left\|x_{1}(t)-x_{2}(t)\right\|_{X} \leq M\left\|\xi_{1}-\xi_{2}\right\|_{X} e^{-(\alpha-M \gamma) t}
$$

This implies that

$$
\begin{aligned}
\left\|\mathcal{G}\left(\xi_{1}\right)-\mathcal{G}\left(\xi_{2}\right)\right\|_{X} & \leq M e^{-\alpha T}\left\|\xi_{1}-\xi_{2}\right\|_{X}+\int_{0}^{T} M e^{-\alpha(T-s)} \gamma\left\|x_{1}(s)-x_{2}(s)\right\|_{X} d s \\
& \leq M e^{-\alpha T}\left\|\xi_{1}-\xi_{2}\right\|_{X}+\int_{0}^{T} M e^{-\alpha(T-s)} \gamma M\left\|\xi_{1}-\xi_{2}\right\|_{X} e^{-(\alpha-M \gamma) s} d s \\
& =M e^{-(\alpha-M \gamma) T}\left\|\xi_{1}-\xi_{2}\right\|_{X} .
\end{aligned}
$$

Then, by the estimations 3.13)-(3.14), one gets the fact that $\mathcal{G}$ has a unique fixed point $x^{*}$ in $X$, i.e., $\mathcal{G}\left(x^{*}\right)=x^{*}$. By the definition of $\mathcal{G}$, there exists a unique mild solution $\bar{x}(t)$ satisfying

$$
\bar{x}(t)=S(t) x^{*}+\int_{0}^{t} S(t-s) G(s, \bar{x}(s)) d s,
$$

and $\bar{x}(0)=\bar{x}(T)=x^{*}$. This fixed point is the initial value from which the mild $T$-periodic solution starts. Then, we define $\overline{\mathbf{x}}$ as follows:

$$
\overline{\mathbf{x}}(t)=\bar{x}(t-k T), \quad t \in[k T,(k+1) T], k=0,1,2, \ldots
$$

and we define $\overline{\mathbf{y}}$ so that

$$
\overline{\mathbf{y}}(t)=\mathbb{V} \mathbb{I}(t, \overline{\mathbf{x}}(t)), \quad t \geq 0,
$$

which yields that $(\overline{\mathbf{x}}, \overline{\mathbf{y}})$ is a mild $T$-periodic solution of $3.1-3.2$.

Remark 3.10. If the semigroup $S(t)$ satisfies $\left(\mathrm{A}^{*}\right)$ with $M=1$, that is,

$$
S(t) \leq e^{-\alpha t}, \quad \forall t \geq 0
$$

Then the conclusion of Theorem 3.9 holds with independent period $T$. In particular, the problem 3.1 -3.2 has a mild $T$-periodic solution provided that

$$
\alpha>a+\frac{b \eta_{1 h}}{\eta_{B}-\eta_{2 h}}
$$




\section{In case of multivalued perturbation}

In this section, we consider a multivalued version of the differential variational inequalities of parabolic-elliptic type. We consider the system as follows:

$$
\begin{gathered}
x^{\prime}(t) \in A x(t)+F(t, x(t), y(t)), \quad t>0, \\
B y(t)+\partial \phi(y(t)) \ni h(t, x(t), y(t)), \quad t \geq 0 .
\end{gathered}
$$

Clearly, in this model, the perturbation $F$ of dynamic system (4.1) is multivalued. The multivalued map $F$ in 4.1 can be seen as a regularization of a discontinuous nonlinearity by the means of Filippov [6]. We will show that under suitable conditions imposed on $F$, the problem (4.1)-4.2 has a mild solution satisfying

$$
x(0)=x_{0},
$$

and under time periodic condition of the multivalued map $F$, we will prove that $4.1-4.2$ has a mild solution satisfying

$$
x(t)=x(t+T)
$$

for some fixed constant time $T>0$.

We need the following assumption imposed on the multivalued map $F$ to get solvability of initial value problem 4.1-4.2.

$(\mathcal{F}) F: \mathbb{R}^{+} \times X \times Y \rightarrow \mathcal{P}(X)$ has nonempty, convex, weakly compact and

(1) For each $(x, y) \in X \times Y, F(\cdot, x, y): \mathbb{R}^{+} \rightarrow \mathcal{P}(X)$ is strongly measurable.

(2) For each $t, F(t, \cdot, \cdot): X \times Y \rightarrow \mathcal{P}(X)$ is upper semicontinuous.

(3) There exist $\bar{a}(\cdot), \bar{b}(\cdot), \bar{c}(\cdot) \in L_{\mathrm{loc}}^{1}\left(\mathbb{R}^{+} ; \mathbb{R}^{+}\right)$such that

$$
\|F(t, x, y)\|:=\sup \{\|\xi\|: \xi \in F(t, x, y)\} \leq \bar{a}(t)\|x\|_{X}+\bar{b}(t)\|y\|_{Y}+\bar{c}(t) .
$$

If $S(t)$ is noncompact, there exist $\bar{p}(\cdot), \bar{q}(\cdot) \in L_{\mathrm{loc}}^{1}\left(\mathbb{R}^{+} ; \mathbb{R}^{+}\right)$such that

$$
\chi_{X} F(t, C, D) \leq \bar{p}(t) \chi_{X}(C)+\bar{q}(t) \chi_{Y}(D) .
$$

Let $\mathcal{S}_{F}$ be a multimap defined by

$$
\begin{gathered}
\mathcal{S}_{F}: C([0, T] ; X) \times L^{1}(0, T ; X) \rightarrow \mathcal{P}\left(L^{1}(0, T ; X)\right), \\
\mathcal{S}_{F}(x, y)=\left\{f \in L^{1}(0, T ; X): f(t) \in F(t, x(t), y(t)) \text { for a.e. } t \in(0, T)\right\} .
\end{gathered}
$$

We have the notion of mild solution to the problem 4.1 -4.2 with initial condition 4.3. 
Definition 4.1. A pair of continuous functions $(x, y)$ for almost everywhere $t \in[0, T]$, is a mild solution of 4.1 -4.2 with condition 4.3 if there exists an integral selection $f \in S_{F}(x, y)$ such that

$$
\begin{cases}x(t)=S(t) x_{0}+\int_{0}^{t} S(t-s) f(s) d s, & t \in[0, T] \\ B y(t)+\partial \phi(y(t)) \ni h(t, x(t), y(t)), & t \in[0, T] .\end{cases}
$$

By similar to the setting of previous section, we can transfer the problem $4.1-4.2$ to the differential inclusion as follows:

$$
x^{\prime}(t) \in A x(t)+F(t, x(t), \mathbb{V} \mathbb{I}(t, x(t))) .
$$

Letting $F(t, x, \mathbb{V} \mathbb{I}(t, x)):=G(t, x), \forall(t, x) \in \mathbb{R}^{+} \times X$, we get

$$
x^{\prime}(t) \in A x(t)+G(t, x(t)) .
$$

Then, $G$ has nonempty weakly compact, convex values. In addition, $G(\cdot, x)$ is strongly measurable for each $x \in X$ and $G(t, \cdot)$ is u.s.c. in $X$. In the sequel, we estimate the sublinear growth of $G$ and the noncompactness of measurable of $G(t, \Omega)$, where $\Omega$ is a bounded subset in $X$. In fact, we have

$$
\begin{aligned}
\|G(t, x)\| & =\sup \left\{\|\xi\|_{X}: \xi \in G(t, x)\right\} \leq \bar{a}(t)\|x\|_{X}+\bar{b}(t)\|\mathbb{V} \mathbb{I}(t, x)\|_{Y}+\bar{c}(t) \\
& \leq \bar{a}(t)\|x\|_{X}+\frac{\bar{b}(t) \eta_{1 h}}{\eta_{B}-\eta_{2 h}}\|x\|_{X}+\|\mathbb{V} \mathbb{I}(t, 0)\|_{Y}+\bar{c}(t) \\
& \leq\left(\bar{a}(t)+\frac{\bar{b}(t) \eta_{1 h}}{\eta_{B}-\eta_{2 h}}\right)\|x\|_{X}+\frac{\eta_{h}(t, 0)}{\eta_{B}-\eta_{2 h}}+\|\mathbb{V} \mathbb{I}(0,0)\|+\bar{c}(t)
\end{aligned}
$$

Denoting

$$
\widehat{a}(t)=\bar{a}(t)+\frac{\bar{b}(t) \eta_{1 h}}{\eta_{B}-\eta_{2 h}}, \quad \widehat{b}(t)=\frac{\eta_{h}(t, 0)}{\eta_{B}-\eta_{2 h}}+\|\mathbb{V} \mathbb{I}(0,0)\|+\bar{c}(t)
$$

then

$$
\|G(t, x)\| \leq \widehat{a}(t)\|x\|_{X}+\widehat{b}(t)
$$

Moreover, if $S(t)$ is noncompact, we have

$$
\chi_{X}(G(t, \Omega)) \leq \bar{p}(t) \chi_{X}(\Omega)+\bar{q}(t) \chi_{Y}(\mathbb{V} \mathbb{I}(t, \Omega)) \leq \bar{p}(t) \chi_{X}(\Omega)+\frac{\bar{q}(t) \eta_{1 h}}{\eta_{B}-\eta_{2 h}} \chi_{X}(\Omega)
$$

So,

$$
\chi_{X}(G(t, \Omega)) \leq \widehat{p}(t) \chi_{X}(\Omega),
$$

where

$$
\widehat{p}(t)=\bar{p}(t)+\frac{\bar{q}(t) \eta_{1 h}}{\eta_{B}-\eta_{2 h}}
$$


We define

$$
\begin{gathered}
\mathcal{P}_{G}: C([0, T] ; X) \rightarrow \mathcal{P}\left(L^{1}(0, T ; X)\right) \\
\mathcal{P}_{G}(x)=\left\{f \in L^{1}(0, T ; X): f(t) \in G(t, x(t)) \text { for a.e. } t \in(0, T)\right\} .
\end{gathered}
$$

Lemma 4.2. Under assumption $(\mathrm{B}),(\mathcal{F})$ and $(\mathrm{H}), \mathcal{P}_{G}$ is well-defined and weakly u.s.c. with weakly compact and convex values. In particular, $\mathcal{P}_{G}$ is a weakly quasicompact multimap.

Proof. The first statement is proved similar to 3 , Theorem 1]. Let $\mathcal{O}$ be a compact subset of $C([0, T] ; X)$. We prove that $\mathcal{P}_{G}(\mathcal{O})$ be a weakly compact subset of $L^{1}(0, T ; X)$.

In fact, let $\left\{f_{n}\right\} \in \mathcal{P}_{G}(\mathcal{O})$, then there exist $x_{n} \in \mathcal{O}$ such that $f_{n}(t) \in G\left(t, x_{n}(t)\right)$, for a.e. $t \in(0, T)$. By the compactness of $\mathcal{O}$, we can assume that $x_{n} \rightarrow x^{*}$ in $C([0, T] ; X)$. By the estimate (4.6), it yields that $\left\{f_{n}\right\}$ is integrably bounded. Moreover, the set $\left\{f_{n}(t)\right\}$ satisfying $f_{n}(t) \in C(t)=G\left(t, \overline{\left\{x_{n}(t)\right\}}\right)$. By using [3, Proposition 2(b)], we obtain that $C(t)$ is weakly compact in $X$. Then, we may assume $f_{n} \rightarrow f^{*}$ in $L^{1}(0, T ; X)$. The proof is complete.

Theorem 4.3. Under assumptions $(\mathrm{A}),(\mathrm{B}),(\mathcal{F})$ and $(\mathrm{H})$, then the initial value problem 4.1) -4.3 has a mild solution on $[0, T]$.

Proof. We introduce the multivalued solution operator

$$
\mathcal{Q}: C([0, T] ; X) \rightarrow \mathcal{P}(C([0, T] ; X)), \quad \mathcal{Q}(x)=\left\{S(t) x_{0}+\mathcal{W}(f): f \in \mathcal{P}_{G}(x)\right\}
$$

By the formulation of $\mathcal{Q}$, we have

$$
\mathcal{Q}(x)=S(t) x_{0}+\mathcal{Q} \circ \mathcal{P}_{G}(x)
$$

For each $x \in C([0, T] ; X), \mathcal{P}_{G}(x)$ is a weakly compact set in $L^{1}(0, T ; X)$. So $\mathcal{W} \circ \mathcal{P}_{G}(x)$ is compact in $C([0, T] ; X)$ due to Proposition 3.5 . In addition, $\mathcal{P}_{G}(x)$ is a convex subset of $X$. That is, the multimap $\mathcal{Q}$ has compact and convex values.

We now show that there exists a nonempty convex subset $\mathcal{M}_{0} \subset C([0, T] ; X)$ such that $\mathcal{F}\left(\mathcal{M}_{0}\right) \subset \mathcal{M}_{0}$. Let $y \in \mathcal{Q}(x)$, then there exists $f \in \mathcal{P}_{G}(x)$ such that

$$
\begin{aligned}
\|y(t)\|_{X} & \leq\left\|S(t) x_{0}\right\|_{X}+\left\|\int_{0}^{t} S(t-s) f(s) d s\right\|_{X} \\
& \leq M\left\|x_{0}\right\|_{X}+\int_{0}^{t}\|S(t-s)\|_{\mathcal{L}(X)}\|f(s)\|_{X} d s \\
& \leq M\left\|x_{0}\right\|_{X}+M \int_{0}^{t}\left[\widehat{a}(s)\|x(s)\|_{X}+\widehat{b}(s)\right] d s .
\end{aligned}
$$


Similar to the proof of Theorem 3.6, we can construct a subset $D \subset X$ such that $D$ is compact and $\mathcal{Q}$ maps $D$ to $\mathcal{P}(D)$.

We now consider $\mathcal{Q}: D \rightarrow \mathcal{P}(D)$. In order to apply the fixed point principle given by Theorem 2.9, it remains to show that $\mathcal{Q}$ has a closed graph. Let $\left\{y_{n}\right\} \subset D$ with $y_{n} \rightarrow y^{*}$ and $z_{n} \in \mathcal{Q}\left(y_{n}\right)$ with $z_{n} \rightarrow z^{*}$. We have

$$
z_{n}(t) \in S(t) x_{0}+\mathcal{W} \circ \mathcal{P}_{G}\left(y_{n}\right)(t)
$$

Let $f_{n} \in \mathcal{P}_{G}\left(y_{n}\right)$ such that

$$
z_{n}(t)=S(t) x_{0}+\mathcal{W}\left(f_{n}\right)(t)
$$

Because $\mathcal{P}_{G}$ is weakly u.s.c., we can employ Lemma 2.7 to state that $f_{n} \rightarrow f^{*}$ in $L^{1}(0, T ; X)$ and $f^{*} \in \mathcal{P}_{G}\left(u^{*}\right)$. Moreover, let $K(t):=G\left(t, \overline{\left\{y_{n}(t)\right\}}\right)$ for a.e. $t \in[0, T]$ with $K(t)$ being compact in $X$ thanks to the fact that $G$ is u.s.c. Taking into account (4.6), we see that $\left\{f_{n}\right\}$ is integrably bounded. Therefore, $\left\{f_{n}\right\}$ is a semicompact sequence. Now, applying Proposition 3.5 yields the compactness of $\left\{\mathcal{W}\left(f_{n}\right)\right\}$ in $C([0, T] ; X)$. Thus, one can pass to the limit in 4.7) to obtain $z^{*}(t)=S(t) x_{0}+\mathcal{W}\left(f^{*}\right)(t)$, where $f^{*} \in \mathcal{P}_{G}\left(y^{*}\right)$. Thus, $z^{*} \in \mathcal{Q}\left(y^{*}\right)$, and the proof is complete.

In the sequel of this section, we present the existence results of problem 4.1 - 4.2. As it is usual in the frame of the abstract Cauchy problem, we consider the following concept of a mild periodic solution of $4.1-4.2$.

Definition 4.4. A pair of continuous functions $(x, y)$ is a mild $T$-periodic solution of (4.1) - 4.2 if (4.4) holds and there exists an integral selection $f \in S_{F}(x, y)$ such that

$$
\begin{cases}x(t)=S(t) x(s)+\int_{s}^{t} S(t-\tau) f(\tau) d \tau & \text { for all } 0 \leq s \leq t \\ B y(t)+\partial \phi(y(t)) \ni h(t, x(t), y(t)), & t \geq 0\end{cases}
$$

We consider the problem as follows:

$$
\begin{cases}x^{\prime}(t)=A x(t)+k(t), & t>0, \\ x(t)=x(t+T), & \forall t \geq 0\end{cases}
$$

thanks to Theorem 3.9, the periodic problem (4.8) has a unique mild solution if $k$ is a $T$-periodic function in $L^{1}(0, \infty ; X)$.

In order to obtain the existence of a mild periodic solution to 4.1 - 4.2 , we replace $(\mathrm{A})$ and $(\mathcal{F})$ by the stronger assumptions:

$\left(\mathcal{A}^{*}\right) A$ satisfies the assumption (A) and $A$ generates a norm continuous, compact semigroup $\{S(t)\}$ in $X$. 
$\left(\mathcal{F}^{*}\right)$ The assumption $(\mathcal{F})$ is satisfied with $\bar{a}(t) \equiv \bar{a}, \bar{b}(t) \equiv \bar{b}$, where $\bar{a}, \bar{b}$ are positive constants, $\bar{c}(\cdot)$ is nonnegative $T$-periodic real-valued. In addition, the multivalued function $F$ is $T$-periodic with respect to the first variable, that is, $F(t+T, x, y)=$ $F(t, x, y), \forall t \geq 0, x \in X, y \in Y$.

The following theorem shows the main result of this section.

Theorem 4.5. Under assumptions $\left(\mathcal{A}^{*}\right),(\mathrm{B}),\left(\mathcal{F}^{*}\right)$ and $\left(\mathrm{H}^{*}\right)$, the problem (4.1)-(4.2) has a mild T-periodic solution provided that $\eta_{B}>\eta_{2 h}$ and

$$
\begin{gathered}
\alpha>M\left(\bar{a}+\frac{\bar{b} \eta_{1 h}}{\eta_{B}-\eta_{2 h}}\right) \\
1-M e^{-\alpha T}-M e^{-(\alpha-M \widehat{a}) T}>0, \text { here } \widehat{a}=\bar{a}+\frac{\bar{b} \eta_{1 h}}{\eta_{B}-\eta_{2 h}} .
\end{gathered}
$$

Proof. First of all, we estimate a priori bound of periodic solution for converted differential inclusion (4.5). Let $x$ be a mild $T$-periodic solution of differential inclusion (4.5), i.e., $x(0)=x(T)$. Then there exists a measurable function $f(t) \in G(t, x(t))$ for a.e. $t \in(0, T)$ such that

$$
x(t)=S(t) x(s)+\int_{s}^{t} S(t-s) f(s) d s .
$$

In particular, we have

$$
x(0)=S(T) x(0)+\int_{0}^{T} S(T-s) f(s) d s .
$$

Because $\{S(t)\}$ is an exponentially stable semigroup, then 1 does not belong to the spectrum of $S(T)$. We get

$$
\begin{aligned}
x(0)=x(T) & =(I-S(T))^{-1} \int_{0}^{T} S(T-s) f(s) d s, \\
\|x(0)\|=\|x(T)\| & \leq\left\|(I-S(T))^{-1} \int_{0}^{T} S(T-s) f(s) d s\right\| \\
& \leq\left\|(I-S(T))^{-1}\right\| \int_{0}^{T} M e^{-\alpha(T-s)}\|f(s)\|_{X} d s \\
& \leq M\left\|(I-S(T))^{-1}\right\| \int_{0}^{T} e^{-\alpha(T-s)}(\widehat{a}\|x(s)\|+\widehat{b}(s)) d s,
\end{aligned}
$$

where $\widehat{a}=\bar{a}+\frac{\bar{b} \eta_{1 h}}{\eta_{B}-\eta_{2 h}}$ and $\widehat{b}$ is determined by the estimate 4.6.

On the other hand, by the integral formula of $x(\cdot)$, we have

$$
\begin{aligned}
\|x(t)\|_{X} & \leq\|S(t)\|\|x(0)\|_{X}+\left\|\int_{0}^{t} S(t-s) f(s) d s\right\|_{X} \\
& \leq M e^{-\alpha t}\|x(0)\|_{X}+M \int_{0}^{t} e^{-\alpha(t-s)}\left(\widehat{a}\|x(s)\|_{X}+\widehat{b}(s)\right) d s \\
& \leq M e^{-\alpha t}\|x(0)\|_{X}+M \int_{0}^{t} e^{-\alpha(t-s)} \widehat{b}(s) d s+M \widehat{a} \int_{0}^{t} e^{-\alpha(t-s)}\|x(s)\|_{X} d s .
\end{aligned}
$$


Then,

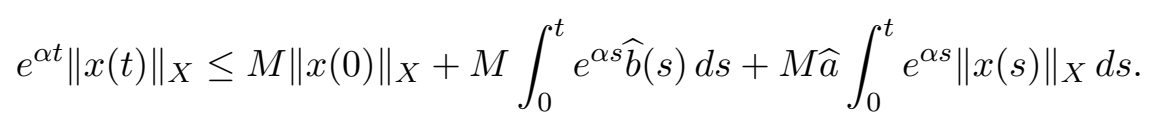

By using the Gronwall inequality, one gets

$$
\begin{aligned}
e^{\alpha t}\|x(t)\| \leq & M\|x(0)\|_{X}+M \int_{0}^{t} e^{\alpha s \widehat{b}}(s) d s \\
& +\int_{0}^{t} M \widehat{a}\left(M\|x(0)\|_{X}+M \int_{0}^{s} e^{\alpha \tau} \widehat{b}(\tau) d \tau\right) e^{M \widehat{a}(t-s)} d s .
\end{aligned}
$$

Suppose that $n T \leq t \leq(n+1) T$, and by using the $T$-periodic property of $\widehat{b}$, one gets the following estimate for the second term of the last right-hand side

$$
\begin{aligned}
& \int_{0}^{t} e^{\alpha s \widehat{b}}(s) d s \leq \int_{0}^{T} e^{\alpha s \widehat{b}}(s) d s+\int_{T}^{2 T} e^{\alpha s \widehat{b}}(s) d s+\cdots+\int_{n T}^{t} e^{\alpha s \widehat{b}}(s) d s \\
& \leq \int_{0}^{T} e^{\alpha s} \widehat{b}(s) d s+e^{\alpha T} \int_{0}^{T} e^{\alpha s} \widehat{b}(s) d s+\cdots+e^{n \alpha T} \int_{0}^{t-n T} e^{\alpha s} \widehat{b}(s) d s \\
& \leq \int_{0}^{T} e^{\alpha s} \widehat{b}(s) d s+e^{\alpha T} \int_{0}^{T} e^{\alpha s} \widehat{b}(s) d s+\cdots+e^{n \alpha T} \int_{0}^{T} e^{\alpha s} \widehat{b}(s) d s \\
& =\left(1+e^{\alpha T}+\cdots+e^{n \alpha T}\right) \int_{0}^{T} e^{\alpha s \widehat{b}(s) d s} \\
& =\frac{e^{(n+1) \alpha T}-1}{e^{\alpha T}-1} \int_{0}^{T} e^{\alpha s} \widehat{b}(s) d s .
\end{aligned}
$$

Hence, for all $t>0$ we get

$$
\begin{aligned}
e^{-\alpha t} \int_{0}^{t} e^{\alpha s \widehat{b}}(s) d s & \leq \frac{e^{((n+1) T-t) \alpha}-e^{-\alpha t}}{e^{\alpha T}-1} \int_{0}^{T} e^{\alpha s \widehat{b}}(s) d s \\
& \leq e^{(n T-t) \alpha} \frac{e^{\alpha T}}{e^{\alpha T}-1} \int_{0}^{T} e^{\alpha s \widehat{b}(s) d s} \\
& <\frac{e^{\alpha T}}{e^{\alpha T}-1} \int_{0}^{T} e^{\alpha s \widehat{b}}(s) d s
\end{aligned}
$$

Then, we have

$$
\begin{aligned}
\|x(t)\|_{X} \leq & M\|x(0)\|_{X} e^{-\alpha t}+M \frac{e^{\alpha T}}{e^{\alpha T}-1} \int_{0}^{T} e^{\alpha s \widehat{b}(s) d s} \\
& +M e^{-(\alpha-M \widehat{a}) t}\|x(0)\|_{X}+M e^{-(\alpha-M \widehat{a}) t} \frac{e^{\alpha T}}{e^{\alpha T}-1} \int_{0}^{T} e^{\alpha s \widehat{b}(s) d s .}
\end{aligned}
$$

Letting $t=T$ and using $x(0)=x(T)$, we obtain

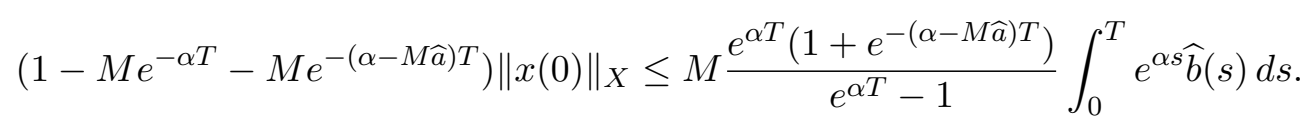


So we arrive at

$$
\|x(0)\|_{X} \leq \mathbf{a}
$$

where

$$
\mathbf{a}=M \frac{e^{\alpha T}\left(1+e^{-(\alpha-M \widehat{a}) T}\right)}{\left(e^{\alpha T}-1\right)\left(1-M e^{-\alpha T}-M e^{-(\alpha-M \widehat{a}) T}\right)} \int_{0}^{T} e^{\alpha s \widehat{b}}(s) d s .
$$

From that, we have an estimate for $\|x(t)\|_{X}$

$$
\|x(t)\|_{X} \leq 2 M \mathbf{a}+2 M \frac{e^{\alpha T}}{e^{\alpha T}-1} \int_{0}^{T} e^{\alpha s} \widehat{b}(s) d s .
$$

By letting $\Theta=\max \left\{\mathbf{a} ; 2 M \mathbf{a}+2 M \frac{e^{\alpha T}}{e^{\alpha T}-1} \int_{0}^{T} e^{\alpha s} \widehat{b}(s) d s\right\}$, we obtain that

$$
\|x(t)\| \leq \Theta, \quad \forall t \in[0, T]
$$

Now, consider the following multifunction

$$
\widehat{G}(t, x)= \begin{cases}G(t, x) & \text { if }\|x\| \leq \Theta \\ G\left(t, \frac{\Theta x}{\|x\|}\right) & \text { if }\|x\|>\Theta\end{cases}
$$

It is easy to see that, the corresponding $(t, x) \rightarrow \widehat{G}(t, x)$ is measurable and $x \rightarrow \widehat{G}(t, x)$ is u.s.c. for each $t \in(0, T)$. Furthermore,

$$
\|\widehat{G}(t, x)\| \leq \widehat{a}(t)+\widehat{b}(t) \Theta:=\Lambda(t)
$$

where $\Lambda \in L^{1}(0, T ; X)$. Let

$$
B(\Lambda)=\left\{h \in L^{1}(0, T ; X):\|h(t)\| \leq \Lambda(t) \text { a.e. } t \in(0, T)\right\}
$$

which is bounded in $L^{1}(0, T ; X)$.

For each $h \in B(\Lambda)$, we consider the problem

$$
\begin{aligned}
x^{\prime}(t) & =A x(t)+h(t), \\
x(t) & =x(t+T), \quad t \geq 0,
\end{aligned}
$$

and let $x(h)(\cdot)$ is a unique mild solution of $4.9-4.10$. We denote

$$
\mathcal{U}=\{x(h)(\cdot): x(h) \text { is a mild solution of } 4.9-4.10, h \in B(\Lambda)\}
$$

We prove that $\mathcal{U}$ is a relatively compact set in $C([0, T] ; X)$ by showing two arguments as follows: 
(1) $\mathcal{U}$ is equicontinuous. In fact, for any $x \in \mathcal{U}$ and $0 \leq t \leq t^{\prime} \leq T$, we have

$$
\begin{aligned}
\left\|x(t)-x\left(t^{\prime}\right)\right\| & \leq\left\|S(t)-S\left(t^{\prime}\right)\right\|\|x(0)\|+\int_{t}^{t^{\prime}}\|S(t-s)\|\|f(s)\| d s \\
& \leq\left\|S(t)-S\left(t^{\prime}\right)\right\| \Theta+M \int_{t}^{t^{\prime}}(\widehat{a}(s)\|x(s)\|+\widehat{b}(s)) d s \\
& \leq\left\|S(t)-S\left(t^{\prime}\right)\right\| \Theta+M \int_{t}^{t^{\prime}}(\widehat{a}(s) \Theta+\widehat{b}(s)) d s .
\end{aligned}
$$

Thus, by the norm-continuity of the semigroup $\{S(t)\}$ and the uniform integrability of $\widehat{a}$, $\widehat{b}$, we deduce that $\mathcal{U}$ is equicontinuous.

(2) $\mathcal{U}(t)$ is relatively compact in $C([0, T] ; X)$.

$\mathcal{U}$ is uniformly bounded. In fact, for any $x \in \mathcal{U}$, we have $\|x(t)\| \leq c_{1}+c_{2}\|\Lambda\|_{1}$ for all $t \in[0, T]$. Moreover, by employing the representation of the mild $T$-periodic solution, one gets

$$
\mathcal{U}(t)=\left\{S(t)(I-S(T))^{-1} \int_{0}^{T} S(T-s) h(s) d s+\int_{0}^{t} S(t-s) h(s) d s: h \in B(\Lambda)\right\} .
$$

Thus, due to the compactness and boundedness of $S(t)$ and $B(\Lambda)$, respectively, we deduce that $\mathcal{U}(t)$ is relatively compact in $X$ for each $t \in[0, T]$.

Therefore, by Arzela-Ascoli theorem, $\mathcal{U}$ is relatively compact.

For any $h \in B(\Lambda)$, we consider the multimap

$\mathcal{G}: B(\Lambda) \rightarrow \mathcal{U}, \quad \mathcal{G}(h)=x, x$ is a unique mild periodic solution of 4.9 - 4.10 .

We show that $\mathcal{G}$ is continuous from $w-B(\Lambda)$ into $\mathcal{U}$. In fact, for any $h_{n}, h_{n} \in B(\Lambda)$ with $h_{n} \rightarrow h$ in $L^{1}(0, T ; X)$, we only need to prove that $\mathcal{G}\left(h_{n}\right) \rightarrow \mathcal{G}(h)$ in $C([0, T] ; X)$. Let $x_{n}=\mathcal{G}\left(h_{n}\right)$ and $x=\mathcal{G}(h)$. Since $x_{n} \in \mathcal{U}$ there exists a subsequence of $\left\{x_{n}\right\}$ (still denoted by $\left.\left\{x_{n}\right\}\right)$ and $\widehat{x}$ such that $x_{n} \rightarrow \widehat{x}$ in $C([0, T] ; X)$. By the definition of $\mathcal{U}$, we have

$$
x_{n}(t)=S(t) x_{n}(s)+\int_{s}^{t} S(t-s) h_{n}(s) d s .
$$

Taking limits to the last equation, we have

$$
\widehat{x}(t)=S(t) \widehat{x}(s)+\int_{s}^{t} S(t-s) h(s) d s .
$$

And obviously,

$$
\widehat{x}(0)=\widehat{x}(T) .
$$

Thus, $\widehat{x}=\mathcal{G}(h)$ and this implies that $\widehat{x} \in \mathcal{U}$, so $\mathcal{G}\left(h_{n}\right) \rightarrow \mathcal{G}(h)$ in $C([0, T] ; X)$. 
Consider the following multimap

$$
\mathcal{P}_{\widehat{G}} \circ \mathcal{G}: B(\Lambda) \rightarrow \mathcal{P}(B(\Lambda)), \quad h \mapsto \mathcal{P}_{\widehat{G}}(\mathcal{G}(h))
$$

By the same argument as in the proof of Lemma 4.2 , we can obtain that $\mathcal{P}_{\widehat{G}}$ is an weakly upper continuous with nonempty convex and weakly compact in $L^{1}(0, T ; X)$. Therefore, $\mathcal{P}_{\widehat{G}} \circ \mathcal{G}$ is a u.s.c. multimap from $B(\Lambda)$ to $w-B(\Lambda)$, and due to Lemma 2.7 again, $\mathcal{P}_{\widehat{G}} \circ \mathcal{G}$ maps a bounded subset to a relatively compact subset in $w-B(\Lambda)$. This yields the compactness of $\mathcal{P}_{\widehat{G}} \circ \mathcal{G}$. Then using Theorem 2.10, there exists a fixed point $\bar{h} \in B(\Lambda)$ such that $\bar{h} \in \mathcal{P}_{\widehat{G}}(\mathcal{G}(\bar{h}))$. Let $\bar{x}=\mathcal{G}(\bar{h})$, by construction of the selection multimap $\mathcal{P}_{\widehat{G}}$, we get $\bar{h}(t) \in \widehat{G}(t, \bar{x}(t))$ a.e. on $(0, T)$. By the definition of $\widehat{G}$, we yield $\bar{h}(t) \in G(t, \bar{x}(t))$ a.e. on $[0, T]$. On the other hand, $\bar{x}$ is a mild periodic solution of 4.9$)$ with $h=\bar{h}$, i.e.,

$$
\bar{x}(t)=S(t) \bar{x}(s)+\int_{s}^{t} S(t-s) \bar{h}(s) d s, \quad \bar{x}(0)=\bar{x}(T) .
$$

Therefore, $\bar{x}$ is a periodic of differential inclusion $(4.5)$. We denote

$$
\bar{y}(t)=\mathbb{V} \mathbb{I}(t, \bar{x}(t))
$$

which implies that $(\bar{x}, \bar{y})$ is a mild solution of generalized differential variational inequality $4.1-4.2$ in $[0, T]$ and satisfies

$$
\bar{x}(0)=\bar{x}(T), \quad \bar{y}(0)=\bar{y}(T) .
$$

By letting

$$
\begin{aligned}
& \mathbf{x}(t)=\bar{x}(t-k T), \quad t \in[k T,(k+1) T], k=0,1,2, \ldots, \\
& \mathbf{y}(t)=\mathbb{V} \mathbb{I}(t, \mathbf{x}(t)), \quad t \geq 0,
\end{aligned}
$$

then $(\mathbf{x}, \mathbf{y})$ is a mild $T$-periodic solution of $4.1-4.2$. The proof is complete.

Remark 4.6. As a special case, we mention the problem $1.1-(1.2)$ in finite dimensional spaces, which was considered systematically by Pang and Stewart in [14. In this situation, $A$ is a bounded linear operator and $e^{t A}$ is a compact semigroup. Moreover, under coercive condition of the operator $-A$, that is, $\langle-A x, x\rangle \geq \alpha\|x\|^{2}, \forall x \in \mathbb{R}^{n}$ for some $\alpha>0$, then $e^{t A}$ is an exponentially stable semigroup. We also obtain the periodic solvability for a such class of differential variational inequalities. Noting that, the considering solutions become classical solutions due to the differentiable property of semigroup $e^{t A}$. As mentioned previously, the system 1.1 - 1.2 considered in Euclidean spaces has been studied recently, with some results related to the long time behaviour in terms of global attractors and decay solutions in [13]; the existence and global bifurcation for periodic solutions in [11]. 


\section{Applications}

5.1. Dynamic systems with obstacle constraints

As an application of our results, we consider the partial differential equation/inclusion mixed an elliptic variational inequality. Let $\Omega \subset \mathbb{R}^{n}$ be a bounded domain with smooth boundary. Consider the following problem

$$
\begin{gathered}
\frac{\partial Z}{\partial t}(t, x)-\Delta_{x} Z(t, x)=f(t, x, Z(t, x), u(t, x)), \\
-\Delta_{x} u(t, x)+\beta(u(t, x)-\psi(x)) \ni h(t, x, Z(t, x), u(t, x))
\end{gathered}
$$

with the periodic condition

$$
Z(t, x)=Z(t+T, x), \quad \forall x \in \Omega, t \in \mathbb{R}^{+},
$$

where $T>0$ is given. The maps $f, h: \Omega \times \mathbb{R} \rightarrow \mathbb{R}$ are continuous functions, $\psi$ is in $H^{2}(\Omega)$ and $\beta: \mathbb{R} \rightarrow 2^{\mathbb{R}}$ is a maximal monotone graph

$$
\beta(r)= \begin{cases}0 & \text { if } r>0, \\ \mathbb{R}^{-} & \text {if } r=0, \\ \emptyset & \text { if } r<0 .\end{cases}
$$

Note that, the elliptic variational inequality $(5.2)$ reads as follows:

$$
\begin{aligned}
-\Delta_{x} u(t, x) & =h(x, Z(t, x)) & & \text { in }\{(t, x) \in Q:=(0, T) \times \Omega: u(t, x) \geq \psi(x)\}, \\
-\Delta_{x} u(t, x) & \geq h(x, Z(t, x)) & & \text { in } Q, \\
u(t, x) & \geq \psi(x), & & \forall(t, x) \in Q,
\end{aligned}
$$

which represents a rigorous and efficient way to treat diffusion problems with a free or moving boundary. A simple physical model for the obstacle problem is that of an elastic membrane that occupies a plane domain $\Omega$ and is limited from below by a rigid obstacle $\psi$ while it is under the pressure of a vertical force field of density $h$. This model is called the obstacle elliptic problem (see [2]).

Let $X=L^{2}(\Omega), Y=H_{0}^{1}(\Omega)$, the norm in $X$ and $Y$ is given by

$$
|u|=\sqrt{\int_{\Omega} u^{2}(x) d x}, \quad u \in L^{2}(\Omega) .
$$

The norm in $H_{0}^{1}(\Omega)$ is given by

$$
\|u\|=\sqrt{\int_{\Omega}|\nabla u(x)|^{2} d x}, \quad u \in H_{0}^{1}(\Omega) .
$$


Define the abstract function

$$
F: \mathbb{R}^{+} \times X \times Y \rightarrow \mathcal{P}(X), \quad F(t, Z, u)=f(t, x, Z(x), u(x)),
$$

and the operator

$$
A=\Delta: D(A) \subset X \rightarrow X, \quad D(A)=\left\{H^{2}(\Omega) \cap H_{0}^{1}(\Omega)\right\} .
$$

Then (5.1) can be reformulated as

$$
Z^{\prime}(t)-A Z(t)=F(t, Z(t), u(t))
$$

where $Z(t) \in X, u(t) \in Y$ such that $Z(t)(x)=Z(t, x)$ and $u(t)(x)=u(t, x)$. It is known in 16 that the semigroup $S(t)$ generated by $A$ is compact and exponentially stable, that is,

$$
\|S(t)\|_{\mathcal{L}(X)} \leq e^{-\lambda_{1} t}
$$

then the assumption $\left(\mathcal{A}^{*}\right)$ is satisfied.

We assume, in addition, that there exist nonnegative functions $a(\cdot), b(\cdot) \in L^{\infty}(\Omega)$ such that

$$
\left|f(t, x, p, q)-f\left(t, x, p^{\prime}, q^{\prime}\right)\right| \leq a(x)\left|p-p^{\prime}\right|+b(x)\left|q-q^{\prime}\right|,
$$

and moreover, we suppose $f(t, x, p, q)=f(t+T, x, p, q)$ for all $t \geq 0, x \in \Omega, p, q \in \mathbb{R}$.

By the setting of function $F$, it is easy to see that $F$ is continuous and

$$
\|F(t, Z, u)-F(t, \bar{Z}, \bar{u})\| \leq\|a\|_{\infty}\|Z-\bar{Z}\|_{X}+\frac{\|b\|_{\infty}}{\sqrt{\lambda_{1}}}\|u-\bar{u}\|_{Y} .
$$

Thus, (F) holds.

Consider the elliptic variational inequality (5.2), putting $B=-\Delta$, where $-\Delta$ stands for the Laplace operator

$$
\langle u,-\Delta v\rangle:=\int_{\Omega} \nabla u(x) \nabla v(x) d x,
$$

then $\langle B u, u\rangle=\|u\|_{U}^{2}$. So, the assumption (B) takes place with $\eta_{B}=1$.

As far as the nonlinear function $h$ is concerned, we assume that the map $h: \mathbb{R}^{+} \times \Omega \times$ $\mathbb{R} \times \mathbb{R} \rightarrow \mathbb{R}$ satisfies $h(t, x, p, q)=h(t+T, x, p, q), \forall x \in \Omega, t \geq 0, p, q \in \mathbb{R}$ and

$$
\left|h(t, x, p, q)-h\left(\bar{t}, x, p^{\prime}, q^{\prime}\right)\right| \leq \eta(t, \bar{t})+c(x)\left|p-p^{\prime}\right|+d(x)\left|q-q^{\prime}\right|, \quad \forall x \in \Omega, p, q \in \mathbb{R},
$$

where $c(\cdot), d(\cdot)$ are the nonnegative functions in $L^{\infty}(\Omega)$ and $\eta(\cdot, \cdot): \mathbb{R}^{+} \times \mathbb{R}^{+} \rightarrow \mathbb{R}^{+}$is a nonnegative continuous function.

Let $h: \mathbb{R}^{+} \times X \times Y \rightarrow L^{2}(\Omega), h(t, \bar{Z}, \bar{u})(x)=h(t, x, \bar{Z}(x), \bar{u}(x))$, we obtain

$$
|h(t, Z, u)-h(\bar{t}, \bar{Z}, \bar{u})| \leq\|c\|_{\infty}\|Z-\bar{Z}\|_{X}+\frac{\|d\|_{\infty}}{\sqrt{\lambda_{1}}}\|u-\bar{u}\|_{Y}+\eta(t, \bar{t})|\Omega| .
$$


Then the EVI (5.2) reads as

$$
B u(t)+\partial I_{K}(u(t)) \ni h(t, Z(t), u(t)),
$$

where

$$
\begin{aligned}
K & =\left\{u \in H_{0}^{1}(\Omega): u(y) \geq \psi(x) \text { for a.e. } x \in \Omega\right\}, \\
\partial I_{K}(u) & =\left\{u \in H_{0}^{1}(\Omega): \int_{\Omega} u(x)(v(x)-z(x)) d x \geq 0, \forall z \in K\right\} \\
& =\left\{u \in H_{0}^{1}(\Omega): u(x) \in \beta(v(x)-\psi(x)) \text { for a.e. } x \in \Omega\right\} .
\end{aligned}
$$

It follows that $(\mathrm{H})$ is testified.

We have the following result due to Theorem 3.9 .

Theorem 5.1. If $\|d\|_{\infty}^{2}<\lambda_{1}$ and

$$
\|a\|_{\infty}+\frac{\|b\|_{\infty}\|c\|_{\infty}}{\sqrt{\lambda_{1}}-\|d\|_{\infty}}<\lambda_{1},
$$

then the problem (5.1)-(5.2) has a unique mild T-periodic solution $(\mathbf{Z}, \mathbf{u})$.

In case of multivalued perturbation, we replace (5.1) with a partial differential inclusion as follows:

$$
\begin{gathered}
\frac{\partial Z}{\partial t}(t, x)-\Delta_{x} Z(t, x)=f(t, x), \\
f(t, x)=\lambda f_{1}(t, x, Z(t, x), u(t, x)) \\
+(1-\lambda) f_{2}(t, x, Z(t, x), u(t, x)), \quad \lambda \in[0,1], t>0, x \in \Omega,
\end{gathered}
$$

where $f_{1}, f_{2}: \mathbb{R}^{+} \times \Omega \times \mathbb{R} \times \mathbb{R} \rightarrow \mathbb{R}$ are $T$-periodic continuous functions with respect to their first variables. Define the multivalued function

$$
\begin{gathered}
F: \mathbb{R}^{+} \times X \times Y \rightarrow \mathcal{P}(X), \\
F(t, \bar{Z}, \bar{u})(x)=\left\{\lambda f_{1}(t, x, \bar{Z}(x), \bar{u}(x))+(1-\lambda) f_{2}(t, x, \bar{Z}(x), \bar{u}(x))\right\} .
\end{gathered}
$$

It is easily seen that $F$ is a multimap with closed convex, compact values and $F(t+T)=$ $F(t), \forall t \geq 0$. Suppose that there exist nonnegative functions $a_{1}(\cdot), a_{2}(\cdot), b_{1}(\cdot), b_{2}(\cdot) \in$ $L^{\infty}(\Omega)$ and $c_{1}(t, \cdot), c_{2}(t, \cdot) \in L^{2}(\Omega)$ for each $t>0$ such that

$$
\begin{aligned}
& \left|f_{1}(t, x, p, q)\right| \leq a_{1}(x)|p|+b_{1}(x)|q|+c_{1}(t, x), \quad \forall t \geq 0, x \in \Omega, p, q \in \mathbb{R}, \\
& \left|f_{2}(t, x, p, q)\right| \leq a_{2}(x)|p|+b_{2}(x)|q|+c_{2}(t, x), \quad \forall t \geq 0, x \in \Omega, p, q \in \mathbb{R} .
\end{aligned}
$$

Because $f_{1}$ and $f_{2}$ are continuous, the fact that $F$ has a closed graph can be testified by a simple argument. Furthermore, if $\left\{\bar{Z}_{n}\right\} \subset X,\left\{\bar{u}_{n}\right\} \subset Y$ are convergent sequences, then 
one can find a sequence $\left\{f_{n}\right\}, f_{n} \in F\left(\cdot, \bar{Z}_{n}, \bar{u}_{n}\right)$ that is convergent in $X$ by using the Lebesgue dominated convergence theorem. So $F$ is quasi-compact. By Lemma 2.6, $F$ is a u.s.c. multimap. Moreover,

$$
\begin{aligned}
\|F(t, \bar{Z}, \bar{u})\| \leq & \max \left\{\left\|a_{1}\right\|_{\infty},\left\|a_{2}\right\|_{\infty}\right\}\|\bar{Z}\|_{X}+\frac{1}{\sqrt{\lambda_{1}}} \max \left\{\left\|b_{1}\right\|_{\infty},\left\|b_{2}\right\|_{\infty}\right\}\|\bar{u}\|_{Y} \\
& +\max \left\{\left\|c_{1}(t)\right\|_{X},\left\|c_{2}(t)\right\|_{X}\right\} .
\end{aligned}
$$

Then, $\left(\mathcal{F}^{*}\right)$ is testified.

Applying Theorem 4.5, we arrive at the last result in this subsection related to the existence of periodic solutions of (5.3)-(5.4) mixed the elliptic variational inequality (5.2).

Theorem 5.2. If $\|d\|_{\infty}^{2}<\lambda_{1}$ and

$$
\gamma:=\lambda_{1}-\max \left\{\left\|a_{1}\right\|_{\infty},\left\|a_{2}\right\|_{\infty}\right\}+\frac{\max \left\{\left\|b_{1}\right\|_{\infty},\left\|b_{2}\right\|_{\infty}\right\}\|c\|_{\infty}}{\sqrt{\lambda_{1}}-\|d\|_{\infty}}>0,
$$

then the problem (5.2)-(5.4) has a mild T-periodic solution $(\mathbf{Z}, \mathbf{u})$ provided that

$$
1-e^{-T}-e^{-\gamma T}>0
$$

\subsection{Lattice differential variational inequalities}

We consider the lattice differential variational inequalities of the following form:

$$
\begin{cases}\frac{d}{d t} Z_{i}(t)=(A Z(t))_{i}+f_{i}\left(t, Z_{i}(t), u_{i}(t)\right), & t>0, \\ -(A u(t))_{i}+\partial \phi_{i}\left(u_{i}(t)\right) \ni h_{i}\left(t, Z_{i}(t), u_{i}(t)\right), & t>0, \\ Z_{i}(t)=Z_{i}(t+T), & \forall t \geq 0,\end{cases}
$$

where $Z=\left(Z_{i}\right):[0, \infty) \rightarrow \ell^{2}, u=\left(u_{i}\right):[0, \infty) \rightarrow \ell^{2}$ are unknown functions, $A: \ell^{2} \rightarrow \ell^{2}$ is a linear operator defined by

$$
(A v)_{i}=v_{i+1}-(2+\lambda) v_{i}+v_{i-1}, \quad v \in \ell^{2}
$$

for some $\lambda>0$, where $\ell^{2}$ is the space of sequences $\left(v_{i}\right)_{i \in \mathbb{Z}}$ satisfying $\sum_{i \in \mathbb{Z}} v_{i}^{2}<\infty$, which becomes a Hilbert space with the inner product $(u, v)_{\ell^{2}}=\sum_{i \in \mathbb{Z}} u_{i} v_{i}$.

Lattice differential systems like (5.5) can be seen as a model of semi-discretization for the differential variational inequality

$$
\begin{cases}\frac{d}{d t} Z(t, x)=\frac{\partial^{2}}{\partial x^{2}} Z(t, x)-\lambda Z(t, x)+f(t, Z(t, x), u(t, x)), & x \in \mathbb{R}, t>0, \\ -\frac{\partial^{2}}{\partial x^{2}} u(t, x)+\lambda u(t, x)+\partial \phi(u(t, x)) \ni h(t, Z(t, x), u(t, x)), & t>0,\end{cases}
$$

where the discretizing is made in spatial variable $x$. Here, $f=\left(f_{i}\right), h=\left(h_{i}\right), \phi=\left(\phi_{i}\right)$. 
Let $\mathcal{B}: \ell^{2} \rightarrow \ell^{2}$ be the linear operator defined by $(\mathcal{B} v)_{i}=v_{i+1}-v_{i}$, then its adjoint operator $\mathcal{B}^{*}$ is given by $\left(\mathcal{B}^{*} v\right)_{i}=v_{i-1}-v_{i}$. In addition, if $\bar{A}: \ell^{2} \rightarrow \ell^{2}$ is the operator defined by $(\bar{A} v)_{i}=v_{i+1}-2 v_{i}+v_{i-1}$ then

$$
-\bar{A}=\mathcal{B B}^{*}=\mathcal{B}^{*} \mathcal{B}
$$

It should be note that $A=\bar{A}-\lambda I$ is a bounded operator on $\ell^{2}$. Then the semigroup $\left\{e^{t A}: t \geq 0\right\}$ is uniformly continuous (see, e.g., 16 ) and hence norm continuous. However, this semigroup is non-compact, since it can be extended to a group $\left\{e^{t A}: t \in \mathbb{R}\right\}$ and the identity operator $I=e^{t A} e^{-t A}$ is noncompact.

To obtain the exponential stability of $\left\{e^{t A}: t \geq 0\right\}$, we consider the system

$$
\frac{d v(t)}{d t}=\bar{A} v(t)-\lambda v(t), \quad v(t) \in \ell^{2} .
$$

Multiplying both sides of the latter equation by $v(t)$, we get

$$
\begin{aligned}
\frac{1}{2} \frac{d}{d t}\|v(t)\|^{2} & =(\bar{A} v(t), v(t))-\lambda\|v(t)\|^{2}=-\left(\mathcal{B}^{*} \mathcal{B} v(t), v(t)\right)-\lambda\|v(t)\|^{2} \\
& =-\|\mathcal{B} v(t)\|^{2}-\lambda\|v(t)\|^{2} \leq-\lambda\|v(t)\|^{2} .
\end{aligned}
$$

Then the Gronwall lemma yields

$$
\|v(t)\| \leq e^{-\lambda t}\|v(0)\|
$$

and, therefore, one has the estimate $\left\|e^{t A}\right\| \leq e^{-\lambda t}, t \geq 0$, then the semigroup $\left\{e^{t A}: t \geq 0\right\}$ is exponentially stable. The assumption $\left(\mathrm{A}^{*}\right)$ is verified.

By setting $B:=-\bar{A}+\lambda I$, one gets

$$
(B u, u) \geq \lambda\|u\|^{2},
$$

which satisfies the assumption (B) with $\eta_{B}=\lambda$.

Now, we give the following assumptions:

(H1) The functions $f_{i}: \mathbb{R}^{+} \times \mathbb{R}^{2} \rightarrow \mathbb{R}, i \in \mathbb{Z}$ are Lipschitz continuous and satisfy

$$
\left|f_{i}(t, x, y)-f_{i}\left(t, x^{\prime}, y^{\prime}\right)\right| \leq a\left(\left|x-x^{\prime}\right|+\left|y-y^{\prime}\right|\right)
$$

for some $a>0, b_{i}: \mathbb{R}^{+} \rightarrow \mathbb{R}^{+}, b(t)=\left(b_{i}(t)\right)_{i \in \mathbb{Z}} \in \ell^{2}$ for all $t \geq 0$. Moreover, $f_{i}, b_{i}$ are $T$-periodic in $t$ for some given $T>0$.

(H2) The functions $h_{i}: \mathbb{R}^{+} \times \mathbb{R}^{2} \rightarrow \mathbb{R}, i \in \mathbb{Z}$ are Lipschitz continuous, i.e., there exist two positive numbers $\zeta_{1}, \zeta_{2}$ and the continuous functions $\eta_{i}, \eta\left(t, t^{\prime}\right)=\left(\eta_{i}\left(t, t^{\prime}\right)\right)_{i \in \mathbb{Z}} \in \ell^{2}$ for all $t, t^{\prime} \geq 0$ such that

$$
\left|h_{i}(t, x, y)-h_{i}\left(t^{\prime}, x^{\prime}, y^{\prime}\right)\right|^{2} \leq \eta_{i}^{2}\left(t, t^{\prime}\right)+\zeta_{1}^{2}\left|x-x^{\prime}\right|^{2}+\zeta_{2}^{2}\left|y-y^{\prime}\right|^{2} .
$$


Let $F, h: \mathbb{R}^{+} \times \ell^{2} \times \ell^{2} \rightarrow \ell^{2}$ be functions defined by

$$
F(t, u, v)=\left(f_{i}\left(t, u_{i}, v_{i}\right)\right)_{i \in \mathbb{Z}}, \quad h(t, u, v)=\left(h_{i}\left(t, u_{i}, v_{i}\right)\right)_{i \in \mathbb{Z}} .
$$

Then, one can check that $f_{1}, f_{2}, h$ are continuous. Furthermore, it follows from (H1), (H2) that

$$
\begin{aligned}
\left\|F(t, u, v)-F\left(t, u^{\prime}, v^{\prime}\right)\right\| & \leq a\left(\left\|u-u^{\prime}\right\|+\left\|v-v^{\prime}\right\|\right), \\
\left\|h(t, u, v)-h\left(t^{\prime}, u^{\prime}, v^{\prime}\right)\right\|^{2} & \leq\left\|\eta\left(t, t^{\prime}\right)\right\|^{2}+\zeta_{1}^{2}\|u\|^{2}+\zeta_{2}^{2}\|v\|^{2},
\end{aligned}
$$

then $(\mathrm{H})$ and $(\mathrm{F})$ are testified.

Now we suppose that

(H3) $\phi_{i}: \mathbb{R} \rightarrow(-\infty ; \infty], i \in \mathbb{Z}$ is proper, lower semicontinuous, convex. Moreover, we assume that

$$
\left(\phi_{i}\left(u_{i}\right)\right)_{i \in \mathbb{Z}} \in \ell^{1}
$$

for all $\left(u_{i}\right)_{i \in \mathbb{Z}} \in \ell^{2}$.

We consider

$$
\phi: \ell^{2} \rightarrow(-\infty, \infty], \quad \phi(u)=\sum_{i \in \mathbb{Z}} \phi_{i}\left(u_{i}\right),
$$

then $\phi$ is a convex, l.s.c., proper function in $\ell^{2}$. Moreover, it is easy to see that $\left(\partial \phi_{i}\right)_{i \in \mathbb{Z}}=$ $\partial \phi$.

The system (5.5) can now be rewritten as

$$
\begin{cases}Z^{\prime}(t)=A Z(t)+F(t, Z(t), u(t)), & t>0, \\ -A u(t)+\partial \phi(t) \ni h(t, Z(t), u(t)), & t>0, \\ Z(t)=Z(t+T) . & \end{cases}
$$

By the above description for (5.5), we can apply Theorem 3.9 to get the existence and uniqueness of time periodic solutions.

Theorem 5.3. Let (H1), (H2) and (H3) hold. Then the problem (5.6) has a unique mild $T$-periodic solution provided that $\zeta_{2}<\lambda$ and

$$
\lambda>a\left(1+\frac{\zeta_{1}}{\lambda-\zeta_{2}}\right) .
$$

\section{Acknowledgments}

The author would like to thank the anonymous referee for the careful reading and useful recommendations. I am deeply thankful to Professor Tran Dinh Ke for introducing to me the differential inclusion theory, giving me a great encouragement and for his helpful suggestions. 


\section{References}

[1] R. R. Akhmerov, M. I. Kamenskiǔ, A. S. Potapov, A. E. Rodkina and B. N. Sadovskiŭ, Measures of Noncompactness and Condensing Operators, Operator Theory: Advances and Applications 55, Birkhäuser Verlag, Basel, 1992.

[2] V. Barbu, Nonlinear Differential Equations of Monotone Types in Banach Spaces, Springer Monographs in Mathematics, Springer, New York, 2010.

[3] D. Bothe, Multivalued perturbations of m-accretive differential inclusions, Israel J. Math. 108 (1998), 109-138.

[4] H. Brézis, Opérateurs maximaux monotones et semi-groupes de contractions dans les espaces de Hilbert, North-Holland Mathematics Studies 5, North-Holland, Amsterdam-London, 1973

[5] X. Chen and Z. Wang, Differential variational inequality approach to dynamic games with shared constraints, Math. Program. 146 (2014), no. 1-2, Ser. A, 379-408.

[6] A. F. Filippov, Differential Equations with Discontinuous Righthand Sides, Mathematics and its Applications (Soviet Series) 18, Kluwer Academic Publishers Group, Dordrecht, 1988.

[7] W Jäger and S. Luckhaus, On explosions of solutions to a system of partial differential equations modelling chemotaxis, Trans. Amer. Math. Soc. 329 (1992), no. 2, 819-824.

[8] Z. Jin and X. Yang, Weak solutions of a parabolic-elliptic type system for image inpainting, ESAIM Control Optim. Calc. Var. 16 (2010), no. 4, 1040-1052.

[9] M. Kamenskii, V. Obukhovskii and P. Zecca, Condensing Multivalued Maps and Semilinear Differential Inclusions in Banach Spaces, De Gruyter Series in Nonlinear Analysis and Applications 7, Walter de Gruyter, Berlin, 2001.

[10] T. D. Ke and D. Lan, Global attractor for a class of functional differential inclusions with Hille-Yosida operators, Nonlinear Anal. 103 (2014), 72-86.

[11] Z. Liu, N. V. Loi and V. Obukhovskii, Existence and global bifurcation of periodic solutions to a class of differential variational inequalities, Internat. J. Bifur. Chaos Appl. Sci. Engrg. 23 (2013), no. 7, 1350125, 10 pp.

[12] A. Nguyen Thi Van and T. D. Ke, Asymptotic behavior of solutions to a class of differential variational inequalities, Ann. Polon. Math. 114 (2015), no. 2, 147-164. 
[13] T. V. A. Nguyen and D. K. Tran, On the differential variational inequalities of parabolic-elliptic type, Math. Methods Appl. Sci. 40 (2017), no. 13, 4683-4695.

[14] J.-S. Pang and D. E. Stewart, Differential variational inequalities, Math. Program. 113 (2008), no. 2, Ser. A, 345-424.

[15] D. E. Stewart, Dynamics with Inequalities: Impacts and hard constraints, Society for Industrial and Applied Mathematics (SIAM), Philadelphia, PA, 2011.

[16] I. I. Vrabie, Compactness Methods for Nonlinear Evolutions, Pitman Monographs and Surveys in Pure and Applied Mathematics 32, John Wiley \& Sons, New York, 1987.

Thi Van Anh Nguyen

Department of Mathematics, Hanoi National University of Education, 136 Xuan Thuy, Cau Giay, Hanoi, Vietnam

E-mail address: anhntv.ktt@hnue.edu.vn 